J. Nonlinear Var. Anal. 6 (2022), No. 1, pp. 89-122

Available online at http://jnva.biemdas.com

https://doi.org/10.23952/jnva.6.2022.1.06

\title{
ACCELERATED INERTIAL SUBGRADIENT EXTRAGRADIENT ALGORITHMS WITH NON-MONOTONIC STEP SIZES FOR EQUILIBRIUM PROBLEMS AND FIXED POINT PROBLEMS
}

\author{
BING TAN ${ }^{1}$, SUN YOUNG CHO ${ }^{2, *}$, JEN-CHIH YAO ${ }^{2}$ \\ ${ }^{1}$ Institute of Fundamental and Frontier Sciences, \\ University of Electronic Science and Technology of China, Chengdu, China \\ ${ }^{2}$ Research Center for Interneural Computing, China Medical University Hospital, \\ China Medical University, Taichung 40402, Taiwan
}

\begin{abstract}
This paper introduces several new accelerated subgradient extragradient methods with inertial effects for approximating a solution of a pseudomonotone equilibrium problem and a fixed point problem involving a quasi-nonexpansive mapping or a demicontractive mapping in real Hilbert spaces. The proposed algorithms use an adaptive non-monotonic step size criterion that does not include any Armijo line search process. Strong convergence theorems of the suggested iterative algorithms are established without the prior knowledge of the Lipschitz constants of the bifunction. Moreover, $R$-linear convergence is guaranteed under the assumption that the bifunction satisfies strong pseudomonotonicity. Applications to variational inequality problems are also considered. Finally, some numerical examples and applications, which demonstrate the advantages and efficiency of the proposed algorithms, are given.
\end{abstract}

Keywords. Equilibrium problem; Fixed point problem; Inertial method; Pseudomonotone bifunction; Subgradient extragradient method.

\section{INTRODUCTION}

The mathematical form of the equilibrium problem (shortly, EP) in the sense of Blum and Oettli [1] is described as follows:

$$
\text { find } x^{*} \in C \text { such that } f\left(x^{*}, y\right) \geq 0, \quad \forall y \in C,
$$

where $C$ is a nonempty, closed, and convex subset of a real Hilbert space $\mathscr{H}$, and $f: \mathscr{H} \times \mathscr{H} \rightarrow$ $\mathbb{R}$ is a given bifunction with $f(x, x)=0$ for all $x \in C$. We denote by $\operatorname{EP}(f)$ the solution set of the problem (EP). Problem (EP) is also well known as the Ky Fan inequality early studied in [2]. Many optimization problems (e.g., variational inequalities, fixed point problems, minimization problems, saddle point problems, and Nash-equilibrium problems) can be unified under the framework of equilibrium problems. In the past decades, many scholars have studied the problem (EP) and proposed a large number of notable methods to solve it; such as the proximal point method [3], the auxiliary problem principle method [4], and the gap function method [5].

\footnotetext{
${ }^{*}$ Corresponding author.

E-mail addresses: bingtan72@gmail.com (B. Tan), ooly61@ hotmail.com (S.Y. Cho), yaojc@ mail.cmu.edu.tw (J.C. Yao).

Received August 28, 2021; Accepted October 3, 2021.
}

(C)2022 Journal of Nonlinear and Variational Analysis 
Next, we review another important issue covered in this paper. Recall that the fixed point problem (shortly, FPP) for a mapping $S: \mathscr{H} \rightarrow \mathscr{H}$ is stated as follows:

$$
\text { find } x^{*} \in \mathscr{H} \text { such that } S x^{*}=x^{*} \text {. }
$$

The solution set of the problem (FPP) is called the fixed point set of $S$ and is denoted by $\mathrm{F}(S)$. Finding a common solution to the equilibrium problem and the fixed point problem in real Hilbert spaces is the concern of this study. That is, we consider finding solutions to the following problem:

$$
\text { find } x^{*} \in C \text { such that } x^{*} \in \mathrm{EP}(f) \cap \mathrm{F}(S) \text {. }
$$

The possibility of applying this common solution problem to a mathematical model whose constraints can be formulated as a fixed-point problem is the motivation and inspiration for its study. This is especially true in practical situations, such as signal processing, network resource allocation, and image recovery. Recently, problem (EPFPP) attracted a great deal of interest from numerous researchers who proposed various approaches to solve it; see, e.g., [6, 7, 8, 9] and the references therein.

In this paper, we focus on extragradient-type methods to solve the problem (EPFPP). The extragradient method was proposed by Korpelevich [10] and further investigated by Quoc et al. [11] for solving equilibrium problems in Euclidean spaces. Recently, based on the ideas of [11], a large number of extragradient-type methods have been proposed for solving equilibrium problems in infinite-dimensional Hilbert spaces; see, e.g., [12, 13, 14, 15] and the references therein. Note that the method introduced by Quoc et al. [11] is a two-step iterative method and requires solving the strongly convex programming problem on the feasible set $C$ twice in each iteration, which affects the computational efficiency of such algorithms when the structure of the feasible set $C$ is complex. To overcome this difficulty, many authors extended the subgradient extragradient method proposed by Censor, Gibali and Reich [16, 17, 18] to solve equilibrium problems in infinite-dimensional Hilbert spaces (see, e.g., [12, 15, 19]). These methods convert the optimization problem on the feasible set in the second step to an optimization problem on a special half-space in each iteration. Thus, the subgradient extragradient type methods improve the computational efficiency of the extragradient-type methods because they only need to solve the optimization problem on the feasible set once. Notice that the step size of the algorithms proposed in the literature $[7,8,9,20]$ is related to the Lipschitz constants of the bifunction $f$. In other words, a prerequisite for these algorithms to work is that they need to know the prior information about the Lipschitz constants of the bifunction $f$. However, this information is usually not easily available in practical applications. Adaptive-type algorithms become particularly important if no prior knowledge of the Lipschitz constants of the bifunction is known. Recently, scholars proposed a number of adaptive extragradient-type algorithms to find common solutions to equilibrium and fixed point problems; see, e.g., [21, 22, 23, 24, 25]. It should be noted that the algorithms presented in $[23,24]$ use the Armijo line search criterion to update the iteration step size. The use of such criterion may add additional computational efforts due to the fact that the strongly convex programming problem on the feasible set may need to be solved several times in each iteration. In addition, the step sizes considered in the algorithms in [22] are decreasing and non-summable, and applying this step size will reduce the convergence speed of the algorithms used. Moreover, the algorithms proposed in [21, 25] apply a new step size criterion that automatically updates the iteration step size by a simple computation 
using some previously known information. However, these algorithms generate a non-increasing sequence of step sizes and will further affect the computational efficiency of such algorithms.

Since the bifunctions in realistic equilibrium problems may be of pseudomonotone. Recently, some efforts have been put into solving pseudomonotone equilibrium problems (see, e.g., $[12,13,14,15])$, which extend some of the results obtained in $[13,22,26]$ for solving monotone (or strongly pseudomonotone) equilibrium problems due to the fact that the pseudomonotone bifunctions contain the monotone bifunctions and strongly pseudomonotone bifunctions. On the other hand, we observe that the fixed point mapping $S$ in $[9,20]$ is demicontractive while the mapping $S$ in $[7,21,25]$ is quasi-nonexpansive or nonexpansive. It is known that the class of demicontractive mappings contains the quasi-nonexpansive mappings and nonexpansive mappings. Therefore, the algorithms proposed in $[9,20]$ have a wider range of applications than the algorithms introduced in [7, 21, 25].

In recent years, a great deal of effort has been devoted to speeding up various algorithms. The inertial term as one of the acceleration techniques has been widely studied by scholars due to its simple form and good acceleration effect. Recall that the inertial idea is based on a discrete version of the second-order dissipative dynamical system (see, e.g., [27, 28] for more details). Recently, many researchers proposed a large number of iterative algorithms to solve equilibrium problems, variational inequalities, splitting problems, monotone inclusion problems, and fixed point problems; see, e.g., [13, 15, 29, 30, 31] and the references therein. A common characteristic of these inertial-type algorithms is that the next iteration depends on the combination of the previous two (or more) iterations. Many numerical experiments and applications have verified that this small change can improve the convergence speed of algorithms without inertial terms.

Inspired and motivated by the above works, in this paper, we introduce three new subgradient extragradient methods with inertial effects to solve pseudomonotone equilibrium problems and fixed point problems in infinite-dimensional Hilbert spaces. The fixed point mapping in the first method is quasi-nonexpansive, and it is demicontractive in the latter two methods. The three methods apply a new non-monotonic step size criterion allowing them to work without the prior knowledge of the Lipschitz constants of the bifunction. Strong convergence theorems of the three proposed algorithms are established under some suitable conditions. Moreover, iterative schemes for solving variational inequality problems are given as a special application of our main results. Finally, we provide some numerical experiments and applications to verify the theoretical results of this paper.

This paper is organized as follows. Some basic definitions and technical lemmas are stated in Section 2. Section 3 introduces three modified inertial subgradient extragradient methods with a new non-monotonic step size criterion for finding common solutions to the equilibrium and the fixed point problems, and analyzes their convergence behavior. In addition, a new subgradient extragradient algorithm for solving the equilibrium problem is proposed and its linear convergence is guaranteed under the assumption of strong pseudomonotonicity. Section 4 considers the applications to the variational inequality problem. Some numerical examples occurring in finite- and infinite-dimensional spaces and applications to optimal control problems are provided in Section 5. Finally, we conclude the paper with a brief summary in Section 6, the last section. 


\section{PRELIMINARIES}

Let $C$ be a nonempty, closed, and convex subset of a real Hilbert space $\mathscr{H}$. The weak convergence and strong convergence of $\left\{x_{n}\right\}$ to $x$ are represented by $x_{n} \rightarrow x$ and $x_{n} \rightarrow x$, respectively. For each $x, y \in \mathscr{H}$ and $\alpha \in \mathbb{R}$, we have the following facts:

$$
\|x+y\|^{2} \leq\|x\|^{2}+2\langle y, x+y\rangle
$$

and

$$
\|\alpha x+(1-\alpha) y\|^{2}=\alpha\|x\|^{2}+(1-\alpha)\|y\|^{2}-\alpha(1-\alpha)\|x-y\|^{2} .
$$

For every point $x \in \mathscr{H}$, there exists a unique nearest point in $C$, denoted by $P_{C}(x)$, such that $P_{C}(x)=\arg \min \{\|x-y\|, y \in C\} . P_{C}$ is called the metric projection of $\mathscr{H}$ onto $C$. It is known that $P_{C}$ has the following property:

$$
\left\langle x-P_{C}(x), y-P_{C}(x)\right\rangle \leq 0, \quad \forall y \in C .
$$

We recall some concepts of monotonicity of a bifunction [1].

Definition 2.1. For all $x, y, z \in C$, a bifunction $f: C \times C \longrightarrow \mathbb{R}$ is said to be:

(i) Lipschitz-type condition on $C$ if there exist two positive constants $c_{1}, c_{2}$ such that $f(x, y)+$ $f(y, z) \geq f(x, z)-c_{1}\|x-y\|^{2}-c_{2}\|y-z\|^{2}$

(ii) monotone on $C$ if $f(x, y)+f(y, x) \leq 0$;

(iii) strongly pseudomonotone on $C$ if there exists a constant $\sigma>0$ such that $f(x, y) \geq 0 \Longrightarrow$ $f(y, x) \leq-\sigma\|x-y\|^{2}$

(iv) pseudomonotone on $C$ if $f(x, y) \geq 0 \Rightarrow f(y, x) \leq 0$.

From the above definitions, it is easy to see that (ii) $\Rightarrow$ (iv) and (iii) $\Rightarrow$ (iv).

Definition 2.2. Assume that $S: \mathscr{H} \rightarrow \mathscr{H}$ is a mapping with $\mathrm{F}(S) \neq \emptyset$. Then

(i) $S$ is called nonexpansive if $\|S x-S y\| \leq\|x-y\|, \forall x, y \in \mathscr{H}$;

(ii) $S$ is called quasi-nonexpansive if $\|S x-y\| \leq\|x-y\|, \forall x \in \mathscr{H}, y \in \mathrm{F}(S)$;

(iii) $S$ is called $\lambda$-demicontractive with $0 \leq \lambda<1$ if

$$
\|S x-z\|^{2} \leq\|x-z\|^{2}+\lambda\|(I-S) x\|^{2}, \quad \forall z \in \mathrm{F}(S), x \in \mathscr{H},
$$

or equivalently

$$
\langle S x-x, x-z\rangle \leq(\lambda-1) / 2 \cdot\|x-S x\|^{2}, \quad \forall z \in \mathrm{F}(S), x \in \mathscr{H},
$$

or equivalently

$$
\langle S x-z, x-z\rangle \leq\|x-z\|^{2}+(\lambda-1) / 2 \cdot\|x-S x\|^{2}, \quad \forall z \in \mathrm{F}(S), x \in \mathscr{H} ;
$$

(iv) $I-S$ is called demiclosed at zero if $\left\{x_{n}\right\} \subset \mathscr{H}, x_{n} \rightarrow x$ and $\left\|S x_{n}-x_{n}\right\| \rightarrow 0$, then $x \in \mathrm{F}(S)$.

In convex analysis, the following definitions are common and important.

Definition 2.3. (i) The subdifferential of a convex function $h: C \rightarrow \mathbb{R}$ at $x \in C$ is given by

$$
\partial h(x):=\{w \in \mathscr{H} \mid h(y)-h(x) \geq\langle w, y-x\rangle, \forall y \in \mathscr{H}\} .
$$

(ii) The subdifferential of a convex function $f(x, \cdot)$ at $y \in \mathscr{H}$ is denoted as

$$
\partial_{2} f(x, y):=\{w \in \mathscr{H} \mid f(x, z)-f(x, y) \geq\langle w, z-y\rangle, \forall z \in \mathscr{H}\} .
$$


(iii) The normal cone $N_{C}$ of $C$ at $x \in C$ is described as

$$
N_{C}(x):=\{w \in \mathscr{H} \mid\langle w, y-x\rangle \leq 0, \forall y \in C\} .
$$

(iv) The proximal operator of a proper, convex, and lower semicontinuous function $g: C \rightarrow$ $(-\infty,+\infty]$ with a positive constant $\lambda>0$ at $x \in \mathscr{H}$ is defined as

$$
\operatorname{prox}_{\lambda g}(x):=\arg \min _{y \in C}\left\{\lambda g(y)+\frac{1}{2}\|x-y\|^{2}\right\} \text {. }
$$

Definition 2.4 ([32]). A sequence $\left\{x_{n}\right\}$ is said to converge $R$-linearly to $x^{*}$ with rate $\eta \in[0,1)$ if there exists a constant $c>0$ such that $\left\|x_{n}-x^{*}\right\| \leq c \eta^{n}, \forall n \in \mathbb{N}$.

The following technical lemmas are important for our convergence analysis.

Lemma 2.1 ([33]). Let $g: C \rightarrow \mathbb{R}$ be a lower semicontinuous and convex subdifferentiable function on $C$. Then, $p$ is a solution of $\min \{g(x): x \in C\}$ if and only if $0 \in \partial g(p)+N_{C}(p)$, where $N_{C}(p)$ and $\partial g(p)$ represents the normal cone of $C$ at $p$ and the subdifferential of $g$ at $p$, respectively.

Lemma 2.2 ([34]). For all $x \in \mathscr{H}, y \in C$, and $\lambda>0$, the following inequality holds:

$$
\lambda\left[g(y)-g\left(\operatorname{prox}_{\lambda g}(x)\right)\right] \geq\left\langle x-\operatorname{prox}_{\lambda g}(x), y-\operatorname{prox}_{\lambda g}(x)\right\rangle .
$$

Lemma 2.3 ([35]). Let $\left\{a_{n}\right\}$ be a sequence of nonnegative real numbers such that there exists a subsequence $\left\{a_{n_{j}}\right\}$ of $\left\{a_{n}\right\}$ such that $a_{n_{j}}<a_{n_{j}+1}$ for all $j \in \mathbb{N}$. Then, there exists a nondecreasing sequence $\left\{m_{k}\right\}$ of $\mathbb{N}$ such that $\lim _{k \rightarrow \infty} m_{k}=\infty$ and the following properties are satisfied by all (sufficiently large) number $k \in \mathbb{N}: a_{m_{k}} \leq a_{m_{k}+1}$ and $a_{k} \leq a_{m_{k}+1}$. In fact, $m_{k}$ is the largest number $n$ in the set $\{1,2, \ldots, k\}$ such that $a_{n}<a_{n+1}$.

Lemma 2.4 ([36]). Let $\left\{a_{n}\right\}$ be a sequence of non-negative real numbers satisfying $a_{n+1} \leq$ $\left(1-\alpha_{n}\right) a_{n}+\alpha_{n} b_{n}, \forall n \geq 0$, where $\left\{\alpha_{n}\right\} \subset(0,1), \sum_{n=0}^{\infty} \alpha_{n}=\infty$ and $\left\{b_{n}\right\}$ is a sequence such that $\limsup _{n \rightarrow \infty} b_{n} \leq 0$. Then, $\lim _{n \rightarrow \infty} a_{n}=0$.

Lemma 2.5 ([37]). Let $S: \mathscr{H} \rightarrow \mathscr{H}$ be a $\beta$-demicontractive with $\mathrm{F}(S) \neq \emptyset$ and let $S_{\lambda}=$ $(1-\lambda) I+\lambda S$, where $\lambda \in(0,1-\beta)$. Then:

(1) $\mathrm{F}(S)=\operatorname{Fix}\left(S_{\lambda}\right)$;

(2) $\left\|S_{\lambda} x-z\right\|^{2} \leq\|x-z\|^{2}-\lambda(1-\beta-\lambda)\|(I-S) x\|^{2}, \forall x \in \mathscr{H}, z \in \mathrm{F}(S)$;

(3) $\mathrm{F}(S)$ is a closed convex subset of $\mathscr{H}$.

\section{Main Results}

In this section, we introduce several new subgradient extragradient methods with inertial effects for finding common solutions to the equilibrium problem and the fixed point problem and analyze their convergence. The proposed algorithms apply some previously known information to generate adaptive non-monotonic step sizes, which allows them to work without the prior information of the Lipschitz constants of the bifunction. Before we start presenting the proposed algorithms, let us assume that the bifunction $f$ satisfies the following conditions:

(A1) $f$ is pseudomonotone on $C$ and $f(x, x)=0$ for all $x \in C$;

(A2) $f$ satisfies the Lipschitz-type condition on $\mathscr{H}$;

(A3) $f(x, \cdot)$ is convex and subdifferentiable on $\mathscr{H}$ for every fixed $x \in \mathscr{H}$;

(A4) $f$ is jointly weakly continuous on $\mathscr{H} \times C$ in the sense that if $x \in \mathscr{H}, y \in C$ and $\left\{x_{n}\right\},\left\{y_{n}\right\}$ converge weakly to $x, y$, respectively, then $\lim _{n \rightarrow \infty} f\left(x_{n}, y_{n}\right) \rightarrow f(x, y)$. 
3.1. The Algorithm 3.1 and its convergence analysis. In this subsection, we introduce a new algorithm to solve the pseudomonotone equilibrium problem and the fixed point problem involving a quasi-nonexpansive mapping. We assume that the proposed Algorithm 3.1 below satisfies the following conditions.

(C1) The solution set of (EPFPP) is nonempty, that is, $\operatorname{EP}(f) \cap \mathrm{F}(S) \neq \emptyset$;

(C2) The bifunction $f$ satisfies the assumptions (A1)-(A4);

(C3) The mapping $S: \mathscr{H} \rightarrow \mathscr{H}$ is quasi-nonexpansive such that $(I-S)$ is demiclosed at zero;

(C4) The mapping $\varphi: \mathscr{H} \rightarrow \mathscr{H}$ is $\rho$-contraction with constant $\rho \in[0,1)$;

(C5) Let $\left\{\varepsilon_{n}\right\}$ and $\left\{\alpha_{n}\right\}$ be two nonnegative sequences such that $\left\{\alpha_{n}\right\} \subset(0,1), \lim _{n \rightarrow \infty} \alpha_{n}=0$, $\sum_{n=1}^{\infty} \alpha_{n}=\infty$ and $\lim _{n \rightarrow \infty} \frac{\varepsilon_{n}}{\alpha_{n}}=0$. Let $\left\{\xi_{n}\right\}$ be a sequence satisfying $\xi_{n}=1+\zeta_{n}$ and $\sum_{n=1}^{\infty} \zeta_{n}<\infty$

(C6) Let $\left\{\beta_{n}\right\}$ be a real sequence such that $\left\{\beta_{n}\right\} \subset(a, b) \subset(0,1)$ for some $b>a>0$.

Remark 3.1. We note here that Condition (C5) is easily satisfied. For example, taking $\alpha_{n}=$ $1 /(n+1), \varepsilon_{n}=1 /(n+1)^{2}$, and $\xi_{n}=1+1 /(n+1)^{1.1}$.

The suggested Algorithm 3.1 is described as follows.

\section{Algorithm 3.1}

Initialization: Take $\theta>0, \lambda_{1}>0, \mu \in(0,1)$ and $\delta \in(0,2 /(1+\mu))$. Let $x_{0}, x_{1} \in \mathscr{H}$.

Iterative Steps: Given the iterates $x_{n-1}$ and $x_{n}(n \geq 1)$. Calculate $x_{n+1}$ as follows:

Step 1. Compute $w_{n}=x_{n}+\theta_{n}\left(x_{n}-x_{n-1}\right)$, where

$$
\theta_{n}= \begin{cases}\min \left\{\frac{\varepsilon_{n}}{\left\|x_{n}-x_{n-1}\right\|}, \theta\right\}, & \text { if } x_{n} \neq x_{n-1} \\ \theta, & \text { otherwise }\end{cases}
$$

Step 2. Compute

$$
y_{n}=\arg \min _{y \in C}\left\{\lambda_{n} f\left(w_{n}, y\right)+\frac{1}{2}\left\|w_{n}-y\right\|^{2}\right\}=\operatorname{prox}_{\lambda_{n} f\left(w_{n}, \cdot\right)}\left(w_{n}\right) .
$$

If $y_{n}=w_{n}=S w_{n}$, then stop and $w_{n} \in \operatorname{EP}(f) \cap \mathrm{F}(S)$. Otherwise, go to Step 3 .

Step 3. Compute

$$
z_{n}=\arg \min _{y \in T_{n}}\left\{\delta \lambda_{n} f\left(y_{n}, y\right)+\frac{1}{2}\left\|w_{n}-y\right\|^{2}\right\}=\operatorname{prox}_{\delta \lambda_{n} f\left(y_{n}, \cdot\right)}\left(w_{n}\right),
$$

where the half-space is defined by

$$
T_{n}=\left\{y \in \mathscr{H}:\left\langle w_{n}-\lambda_{n} v_{n}-y_{n}, y-y_{n}\right\rangle \leq 0\right\} \text { and } v_{n} \in \partial_{2} f\left(w_{n}, y_{n}\right) .
$$

Step 4. Compute $x_{n+1}=\beta_{n} z_{n}+\left(1-\beta_{n}\right) S t_{n}$, where $t_{n}=\alpha_{n} \varphi\left(x_{n}\right)+\left(1-\alpha_{n}\right) z_{n}$ and update the next step size $\lambda_{n+1}$ by

$$
\lambda_{n+1}=\left\{\begin{array}{l}
\min \left\{\frac{\mu\left(\left\|w_{n}-y_{n}\right\|^{2}+\left\|z_{n}-y_{n}\right\|^{2}\right)}{2\left[f\left(w_{n}, z_{n}\right)-f\left(w_{n}, y_{n}\right)-f\left(y_{n}, z_{n}\right)\right]}, \xi_{n} \lambda_{n}\right\}, \\
\text { if } f\left(w_{n}, z_{n}\right)-f\left(w_{n}, y_{n}\right)-f\left(y_{n}, z_{n}\right)>0 \\
\xi_{n} \lambda_{n}, \text { otherwise. }
\end{array}\right.
$$

Set $n:=n+1$ and go to Step 1 . 
Remark 3.2. From Algorithm 3.1, we have the following observations.

(i) It follows from (3.1) that $\lim _{n \rightarrow \infty} \frac{\theta_{n}}{\alpha_{n}}\left\|x_{n}-x_{n-1}\right\|=0$. Indeed, one has $\theta_{n}\left\|x_{n}-x_{n-1}\right\| \leq \varepsilon_{n}$ for all $n \geq 1$, which together with $\lim _{n \rightarrow \infty} \frac{\varepsilon_{n}}{\alpha_{n}}=0$ implies that

$$
\lim _{n \rightarrow \infty}\left(\theta_{n}\left\|x_{n}-x_{n-1}\right\|\right) / \alpha_{n} \leq \lim _{n \rightarrow \infty} \varepsilon_{n} / \alpha_{n}=0 .
$$

(ii) Note that the domain of $z_{n}$ is $T_{n}$. The optimization procedure in Step 3 of Algorithm 3.1 can be efficiently solved by existing methods of convex quadratic programming (see, e.g., [38]), which improves the computational efficiency of the second step of the extragradient method [11], especially when feasible set $C$ and bifunction $f$ have a complex structure.

(iii) It should be pointed out that $v_{n}$ exists and $C \subseteq T_{n}$. With the help of the definition of $y_{n}$ and Lemma 2.1, we have

$$
0 \in \partial_{2}\left(\lambda_{n} f\left(w_{n}, y\right)+\frac{1}{2}\left\|w_{n}-y\right\|^{2}\right)\left(y_{n}\right)+N_{C}\left(y_{n}\right)
$$

where $\partial_{2} f(x, y)$ denotes the subdifferential of $f(x, \cdot)$ at $y$. Therefore, there exists $v_{n} \in$ $\partial_{2} f\left(w_{n}, y_{n}\right)$ and $v \in N_{C}\left(y_{n}\right)$ such that $\lambda_{n} v_{n}+y_{n}-w_{n}+v=0$. According to $v \in N_{C}\left(y_{n}\right)$ and the definition of $N_{C}\left(y_{n}\right)$, one obtains $\left\langle v, y-y_{n}\right\rangle \leq 0$ for all $y \in C$. Hence,

$$
\begin{aligned}
\left\langle w_{n}-y_{n}, y-y_{n}\right\rangle & =\lambda_{n}\left\langle v_{n}, y-y_{n}\right\rangle+\left\langle v, y-y_{n}\right\rangle \\
& \leq \lambda_{n}\left\langle v_{n}, y-y_{n}\right\rangle, \forall y \in C .
\end{aligned}
$$

That is, $\left\langle w_{n}-\lambda_{n} v_{n}-y_{n}, y-y_{n}\right\rangle \leq 0, \forall y \in C$. Consequently, $C \subseteq T_{n}$.

(iv) We show that $w_{n} \in \operatorname{EP}(f) \cap \mathrm{F}(S)$ if $y_{n}=w_{n}=S w_{n}$. From $y_{n}=\operatorname{prox}_{\lambda_{n} f\left(w_{n}, \cdot\right)}\left(w_{n}\right)$ and Lemma 2.2, we obtain

$$
\lambda_{n}\left(f\left(w_{n}, y\right)-f\left(w_{n}, y_{n}\right)\right) \geq\left\langle w_{n}-y_{n}, y-y_{n}\right\rangle, \quad \forall y \in C .
$$

If $w_{n}=y_{n}$ for some $n \in \mathbb{N}$, then $f\left(w_{n}, y\right) \geq 0$ for all $y \in C$ and hence $w_{n} \in \operatorname{EP}(f)$. This together with $w_{n}=S w_{n}$ implies that $w_{n} \in \mathrm{EP}(f) \cap \mathrm{F}(S)$.

Lemma 3.1. Let $\left\{\lambda_{n}\right\}$ be a sequence defined in (3.3). Then $\lim _{n \rightarrow \infty} \lambda_{n}$ exists.

Proof. Since $f$ satisfies the Lipschitz-type condition with constants $c_{1}$ and $c_{2}$, one has

$$
\begin{aligned}
\frac{\mu\left(\left\|w_{n}-y_{n}\right\|^{2}+\left\|z_{n}-y_{n}\right\|^{2}\right)}{2\left[f\left(w_{n}, z_{n}\right)-f\left(w_{n}, y_{n}\right)-f\left(y_{n}, z_{n}\right)\right]} & \geq \frac{\mu\left(\left\|w_{n}-y_{n}\right\|^{2}+\left\|z_{n}-y_{n}\right\|^{2}\right)}{2\left[c_{1}\left\|w_{n}-y_{n}\right\|^{2}+c_{2}\left\|y_{n}-z_{n}\right\|^{2}\right]} \\
& \geq \frac{\mu}{2 \max \left\{c_{1}, c_{2}\right\}} .
\end{aligned}
$$

Thus, $\lambda_{n} \geq \min \left\{\frac{\mu}{2 \max \left\{c_{1}, c_{2}\right\}}, \lambda_{1}\right\}$. From the inequality $(1+x) \leq \mathrm{e}^{x}, \forall x \in \mathbb{R}$ and the definition of $\lambda_{n+1}$, we have

$$
\begin{aligned}
\lambda_{n+1} & \leq \xi_{n} \lambda_{n}=\left(1+\zeta_{n}\right) \lambda_{n} \leq\left(1+\zeta_{n}\right)\left(1+\zeta_{n-1}\right) \lambda_{n-1} \\
& \leq \cdots \leq \prod_{j=1}^{n}\left(1+\zeta_{j}\right) \lambda_{1} \leq \lambda_{1} \prod_{j=1}^{n} \mathrm{e}^{\zeta_{j}} \\
& =\lambda_{1} \mathrm{e}^{\sum_{j=1}^{n} \zeta_{j}} \leq \lambda_{1} \mathrm{e}^{\sum_{j=1}^{\infty} \zeta_{j}}=K<\infty .
\end{aligned}
$$

This yields that $\left\{\lambda_{n}\right\}$ is bounded and $\lambda_{n} \in\left[\min \left\{\frac{\mu}{2 \max \left\{c_{1}, c_{2}\right\}}, \lambda_{1}\right\}, K\right]$. Moreover, one sees that

$$
\lambda_{n+1} \leq\left(1+\zeta_{n}\right) \lambda_{n} \leq \lambda_{n}+\zeta_{n} K, \quad \forall n \geq 1 \text {. }
$$


Consequently,

$$
\lambda_{n+m+1} \leq \lambda_{n+m}+\zeta_{n+m} K \leq \cdots \leq \lambda_{n}+\sum_{j=n}^{n+m} \zeta_{j} K, \quad \forall n, m \geq 1
$$

Therefore, $\varlimsup_{m \rightarrow \infty} \lambda_{m} \leq \lambda_{n}+\sum_{j=n}^{\infty} \zeta_{j} K$, which implies that $\varlimsup_{m \rightarrow \infty} \lambda_{m} \leq \underline{\lim }_{n \rightarrow \infty} \lambda_{n}$. That is, $\lim _{n \rightarrow \infty} \lambda_{n}$ exists. The proof is completed.

Lemma 3.2. Assume that the bifunction $f$ satisfies Assumptions (A1)-(A4). Let $\left\{w_{n}\right\},\left\{y_{n}\right\}$, and $\left\{z_{n}\right\}$ be three sequences generated by Algorithm 3.1. Then, for all $p \in \operatorname{EP}(f) \cap \mathrm{F}(S)$,

$$
\left\|z_{n}-p\right\|^{2} \leq\left\|w_{n}-p\right\|^{2}-\delta_{n}^{*}\left(\left\|w_{n}-y_{n}\right\|^{2}+\left\|z_{n}-y_{n}\right\|^{2}\right),
$$

where $\delta_{n}^{*}=2-\delta-\frac{\delta \mu \lambda_{n}}{\lambda_{n+1}}$ if $\delta \in[1,2 /(1+\mu))$, and $\delta_{n}^{*}=\delta-\frac{\delta \mu \lambda_{n}}{\lambda_{n+1}}$ if $\delta \in(0,1)$.

Proof. According to the definition of $z_{n}$ and Lemma 2.1, one sees that

$$
0 \in \partial_{2}\left(\delta \lambda_{n} f\left(y_{n}, y\right)+\frac{1}{2}\left\|w_{n}-y\right\|^{2}\right)\left(z_{n}\right)+N_{T_{n}}\left(z_{n}\right) .
$$

Therefore, there exist $q_{n} \in \partial_{2} f\left(y_{n}, z_{n}\right)$ and $q \in N_{T_{n}}\left(z_{n}\right)$ satisfying $\delta \lambda_{n} q_{n}+z_{n}-w_{n}+q=0$, which together with the definition of $N_{T_{n}}\left(z_{n}\right)$ yields that

$$
\begin{aligned}
\left\langle w_{n}-z_{n}, y-z_{n}\right\rangle & =\delta \lambda_{n}\left\langle q_{n}, y-z_{n}\right\rangle+\left\langle q, y-z_{n}\right\rangle \\
& \leq \delta \lambda_{n}\left\langle q_{n}, y-z_{n}\right\rangle, \forall y \in T_{n} .
\end{aligned}
$$

From the definition of the subdifferential and $q_{n} \in \partial_{2} f\left(y_{n}, z_{n}\right)$, one has

$$
f\left(y_{n}, y\right)-f\left(y_{n}, z_{n}\right) \geq\left\langle q_{n}, y-z_{n}\right\rangle, \quad \forall y \in C .
$$

It follows from (3.4) and (3.5) that

$$
\delta \lambda_{n}\left(f\left(y_{n}, y\right)-f\left(y_{n}, z_{n}\right)\right) \geq\left\langle w_{n}-z_{n}, y-z_{n}\right\rangle, \quad \forall y \in T_{n} .
$$

From Remark 3.2 (iii), one sees that $\operatorname{EP}(f) \cap \mathrm{F}(S) \subseteq \operatorname{EP}(f) \subseteq C \subseteq T_{n}$. Let $p \in \operatorname{EP}(f) \cap \mathrm{F}(S)$. Substituting $y=p$ in (3.6), we obtain

$$
\delta \lambda_{n}\left(f\left(y_{n}, p\right)-f\left(y_{n}, z_{n}\right)\right) \geq\left\langle w_{n}-z_{n}, p-z_{n}\right\rangle .
$$

In view of $p \in \operatorname{EP}(f)$ and $y_{n} \in C$, one obtains $f\left(p, y_{n}\right) \geq 0$. Moreover, we obtain $f\left(y_{n}, p\right) \leq 0$ by means of the pseudomonotonicity of $f$. From (3.7), we obtain

$$
-\delta \lambda_{n} f\left(y_{n}, z_{n}\right) \geq\left\langle w_{n}-z_{n}, p-z_{n}\right\rangle .
$$

Using the definition of the subdifferential and $v_{n} \in \partial_{2} f\left(w_{n}, y_{n}\right)$, one infers that

$$
f\left(w_{n}, y\right)-f\left(w_{n}, y_{n}\right) \geq\left\langle v_{n}, y-y_{n}\right\rangle, \quad \forall y \in C .
$$

In particular, substituting $y=z_{n}$ in (3.9), one has

$$
f\left(w_{n}, z_{n}\right)-f\left(w_{n}, y_{n}\right) \geq\left\langle v_{n}, z_{n}-y_{n}\right\rangle .
$$

From the definition of $T_{n}$ and $z_{n} \in T_{n}$, one obtains $\left\langle w_{n}-\lambda_{n} v_{n}-y_{n}, z_{n}-y_{n}\right\rangle \leq 0$, which is equivalent to $\left\langle w_{n}-y_{n}, z_{n}-y_{n}\right\rangle \leq \lambda_{n}\left\langle v_{n}, z_{n}-y_{n}\right\rangle$. This together with (3.10) indicates that

$$
\lambda_{n}\left(f\left(w_{n}, z_{n}\right)-f\left(w_{n}, y_{n}\right)\right) \geq\left\langle w_{n}-y_{n}, z_{n}-y_{n}\right\rangle .
$$


Combining (3.8) and (3.11), we obtain

$$
\begin{aligned}
& 2 \delta \lambda_{n}\left(f\left(w_{n}, z_{n}\right)-f\left(w_{n}, y_{n}\right)-f\left(y_{n}, z_{n}\right)\right) \\
\geq & 2 \delta\left\langle w_{n}-y_{n}, z_{n}-y_{n}\right\rangle+2\left\langle w_{n}-z_{n}, p-z_{n}\right\rangle \\
= & \delta\left\|w_{n}-y_{n}\right\|^{2}+\delta\left\|y_{n}-z_{n}\right\|^{2}-\delta\left\|w_{n}-z_{n}\right\|^{2}+\left\|w_{n}-z_{n}\right\|^{2}+\left\|z_{n}-p\right\|^{2}-\left\|w_{n}-p\right\|^{2} .
\end{aligned}
$$

From (3.12) and the definition of $\lambda_{n}$, we can show that

$$
\begin{aligned}
\left\|z_{n}-p\right\|^{2} \leq & \left\|w_{n}-p\right\|^{2}-\delta\left\|w_{n}-y_{n}\right\|^{2}-\delta\left\|y_{n}-z_{n}\right\|^{2}-(1-\delta)\left\|w_{n}-z_{n}\right\|^{2} \\
& +2 \delta \lambda_{n}\left(f\left(w_{n}, z_{n}\right)-f\left(w_{n}, y_{n}\right)-f\left(y_{n}, z_{n}\right)\right) \\
\leq & \left\|w_{n}-p\right\|^{2}-\delta\left\|w_{n}-y_{n}\right\|^{2}-\delta\left\|y_{n}-z_{n}\right\|^{2}-(1-\delta)\left\|w_{n}-z_{n}\right\|^{2} \\
& +\frac{\delta \mu \lambda_{n}}{\lambda_{n+1}}\left(\left\|w_{n}-y_{n}\right\|^{2}+\left\|z_{n}-y_{n}\right\|^{2}\right) .
\end{aligned}
$$

That is,

$$
\begin{aligned}
\left\|z_{n}-p\right\|^{2} \leq & \left\|w_{n}-p\right\|^{2}-(1-\delta)\left\|w_{n}-z_{n}\right\|^{2} \\
& -\delta\left(1-\frac{\mu \lambda_{n}}{\lambda_{n+1}}\right)\left(\left\|w_{n}-y_{n}\right\|^{2}+\left\|z_{n}-y_{n}\right\|^{2}\right) .
\end{aligned}
$$

Note that

$$
\left\|w_{n}-z_{n}\right\|^{2} \leq\left(\left\|w_{n}-y_{n}\right\|+\left\|y_{n}-z_{n}\right\|\right)^{2} \leq 2\left(\left\|w_{n}-y_{n}\right\|^{2}+\left\|z_{n}-y_{n}\right\|^{2}\right),
$$

which yields that

$$
-(1-\delta)\left\|w_{n}-z_{n}\right\|^{2} \leq-2(1-\delta)\left(\left\|w_{n}-y_{n}\right\|^{2}+\left\|z_{n}-y_{n}\right\|^{2}\right), \forall \delta \geq 1 .
$$

This together with (3.13) yields

$$
\left\|z_{n}-p\right\|^{2} \leq\left\|w_{n}-p\right\|^{2}-\left(2-\delta-\frac{\delta \mu \lambda_{n}}{\lambda_{n+1}}\right)\left(\left\|w_{n}-y_{n}\right\|^{2}+\left\|z_{n}-y_{n}\right\|^{2}\right), \forall \delta \geq 1 .
$$

On the other hand, if $\delta \in(0,1)$, then

$$
\left\|z_{n}-p\right\|^{2} \leq\left\|w_{n}-p\right\|^{2}-\delta\left(1-\frac{\mu \lambda_{n}}{\lambda_{n+1}}\right)\left(\left\|w_{n}-y_{n}\right\|^{2}+\left\|z_{n}-y_{n}\right\|^{2}\right), \quad \forall \delta \in(0,1) .
$$

This completes the proof of the lemma.

Remark 3.3. From Lemma 3.1 and the assumptions on $\mu, \delta$ (i.e., $\mu \in(0,1)$ and $\delta \in(0,2 /(1+$ $\mu))$ ), one sees that $\lim _{n \rightarrow \infty} \delta_{n}^{*}=2-\delta-\delta \mu>0$ when $\delta \in[1,2 /(1+\mu))$ and $\lim _{n \rightarrow \infty} \delta_{n}^{*}=$ $\delta-\delta \mu>0$ when $\delta \in(0,1)$. Thus we conclude that $\lim _{n \rightarrow \infty} \delta_{n}^{*}>0$. Moreover, there exists $n_{0}>0$ such that $\delta_{n}^{*}>0$ for all $n \geq n_{0}$.

Theorem 3.1. Assume that Conditions $(C 1)-(C 6)$ hold. Then the sequence $\left\{x_{n}\right\}$ generated by Algorithm 3.1 converges to $p \in \mathrm{EP}(f) \cap \mathrm{F}(S)$ in norm, where $p=P_{\mathrm{EP}(f) \cap \mathrm{F}(S)}(\varphi(p))$.

Proof. Since $f$ satisfies Assumptions (A1)-(A4), we have that the solution set $\operatorname{EP}(f)$ is convex and closed (see [11]). Moreover, $\mathrm{F}(S)$ is also closed and convex (see [39, Proposition 1]). Hence, $P_{\mathrm{EP}(f) \cap \mathrm{F}(S)}(\varphi): \mathscr{H} \rightarrow \mathscr{H}$ is a contraction mapping. From the Banach Contraction Principle, there exists a unique point $p \in \mathscr{H}$ such that $p=P_{\operatorname{EP}(f) \cap \mathrm{F}(S)}(\varphi(p))$. In particular, $p \in \mathrm{EP}(f) \cap \mathrm{F}(S)$. It follows from (2.3) that

$$
\langle\varphi(p)-p, z-p\rangle \leq 0, \quad \forall z \in \mathrm{EP}(f) \cap \mathrm{F}(S) .
$$


By Lemma 3.2 and Remark 3.3, we obtain

$$
\left\|z_{n}-p\right\| \leq\left\|w_{n}-p\right\|, \quad \forall n \geq n_{0} .
$$

For convenience, we divide the proof into four steps.

Claim 1. The sequence $\left\{x_{n}\right\}$ is bounded. By the definition of $w_{n}$, one has

$$
\begin{aligned}
\left\|w_{n}-p\right\| & =\left\|x_{n}+\theta_{n}\left(x_{n}-x_{n-1}\right)-p\right\| \\
& \leq\left\|x_{n}-p\right\|+\alpha_{n} \cdot \frac{\theta_{n}}{\alpha_{n}}\left\|x_{n}-x_{n-1}\right\| .
\end{aligned}
$$

From Remark 3.2 (i), one obtains $\frac{\theta_{n}}{\alpha_{n}}\left\|x_{n}-x_{n-1}\right\| \rightarrow 0$ as $n \rightarrow \infty$. Thus, there exists a constant $M_{1}>0$ such that

$$
\frac{\theta_{n}}{\alpha_{n}}\left\|x_{n}-x_{n-1}\right\| \leq M_{1}, \quad \forall n \geq 1 .
$$

Using (3.15), (3.16), and (3.17), one has

$$
\left\|z_{n}-p\right\| \leq\left\|w_{n}-p\right\| \leq\left\|x_{n}-p\right\|+\alpha_{n} M_{1}, \quad \forall n \geq n_{0} .
$$

Let $\Delta_{n}=\alpha_{n}\left(1-\beta_{n}\right)$. By the definition of $x_{n+1}$ and (3.18), we have

$$
\begin{aligned}
\left\|x_{n+1}-p\right\| & \leq \beta_{n}\left\|z_{n}-p\right\|+\left(1-\beta_{n}\right)\left\|S t_{n}-p\right\| \\
& \leq \beta_{n}\left\|z_{n}-p\right\|+\left(1-\beta_{n}\right)\left\|\alpha_{n} \varphi\left(x_{n}\right)+\left(1-\alpha_{n}\right) z_{n}-p\right\| \\
& \leq \beta_{n}\left\|z_{n}-p\right\|+\left(1-\beta_{n}\right)\left(\alpha_{n}\left\|\varphi\left(x_{n}\right)-\varphi(p)\right\|+\alpha_{n}\|\varphi(p)-p\|+\left(1-\alpha_{n}\right)\left\|z_{n}-p\right\|\right) \\
& \leq\left(1-\Delta_{n}\right)\left\|z_{n}-p\right\|+\rho \Delta_{n}\left\|x_{n}-p\right\|+\Delta_{n}\|\varphi(p)-p\| \\
& \leq\left(1-\Delta_{n}\right)\left\|x_{n}-p\right\|+\rho \Delta_{n}\left\|x_{n}-p\right\|+\Delta_{n}\|\varphi(p)-p\|+\alpha_{n} M_{1} \\
& \leq\left(1-(1-\rho) \Delta_{n}\right)\left\|x_{n}-p\right\|+(1-\rho) \Delta_{n}\left[\frac{\|\varphi(p)-p\|}{1-\rho}+\frac{\alpha_{n} M_{1}}{(1-\rho) \Delta_{n}}\right] \\
& \leq \max \left\{\left\|x_{n}-p\right\|, \frac{\|\varphi(p)-p\|}{1-\rho}+\frac{M_{1}}{(1-\rho)\left(1-\beta_{n}\right)}\right\} \\
& \leq \cdots \leq \max \left\{\left\|x_{n_{0}}-p\right\|, \frac{\|\varphi(p)-p\|}{1-\rho}+\frac{M_{1}}{(1-\rho)\left(1-\beta_{n}\right)}\right\} .
\end{aligned}
$$

This implies that the sequence $\left\{x_{n}\right\}$ is bounded. So the sequences $\left\{\varphi\left(x_{n}\right)\right\}\left\{y_{n}\right\},\left\{z_{n}\right\}$, and $\left\{t_{n}\right\}$ are also bounded.

\section{Claim 2.}

$$
\begin{aligned}
& \delta_{n}^{*}\left(\left\|y_{n}-w_{n}\right\|^{2}+\left\|y_{n}-z_{n}\right\|^{2}\right)+\beta_{n}\left(1-\beta_{n}\right)\left\|S t_{n}-z_{n}\right\|^{2} \\
\leq & \left\|x_{n}-p\right\|^{2}-\left\|x_{n+1}-p\right\|^{2}+2 \alpha_{n}\left(1-\beta_{n}\right)\left\langle\varphi\left(x_{n}\right)-p, t_{n}-p\right\rangle+\alpha_{n} M_{2}
\end{aligned}
$$

for some $M_{2}>0$. Indeed, it follows from (3.18) that

$$
\begin{aligned}
\left\|w_{n}-p\right\|^{2} & \leq\left(\left\|x_{n}-p\right\|+\alpha_{n} M_{1}\right)^{2} \\
& =\left\|x_{n}-p\right\|^{2}+\alpha_{n}\left(2 M_{1}\left\|x_{n}-p\right\|+\alpha_{n} M_{1}^{2}\right) \\
& \leq\left\|x_{n}-p\right\|^{2}+\alpha_{n} M_{2}
\end{aligned}
$$


for some $M_{2}:=\sup _{n \in \mathbb{N}}\left\{2 M_{1}\left\|x_{n}-p\right\|+\alpha_{n} M_{1}^{2}\right\}>0$. Using the definitions of $x_{n+1}$ and $t_{n},(2.1)$, and (2.2), we obtain

$$
\begin{aligned}
& \left\|x_{n+1}-p\right\|^{2}=\left\|\beta_{n} z_{n}+\left(1-\beta_{n}\right) S t_{n}-p\right\|^{2} \\
= & \beta_{n}\left\|z_{n}-p\right\|^{2}+\left(1-\beta_{n}\right)\left\|S t_{n}-p\right\|^{2}-\beta_{n}\left(1-\beta_{n}\right)\left\|S t_{n}-z_{n}\right\|^{2} \\
\leq & \beta_{n}\left\|z_{n}-p\right\|^{2}+\left(1-\beta_{n}\right)\left\|\alpha_{n} \varphi\left(x_{n}\right)+\left(1-\alpha_{n}\right) z_{n}-p\right\|^{2}-\beta_{n}\left(1-\beta_{n}\right)\left\|S t_{n}-z_{n}\right\|^{2} \\
\leq & \beta_{n}\left\|z_{n}-p\right\|^{2}-\beta_{n}\left(1-\beta_{n}\right)\left\|S t_{n}-z_{n}\right\|^{2}+\left(1-\beta_{n}\right)\left(\left(1-\alpha_{n}\right)\left\|z_{n}-p\right\|^{2}\right. \\
& \left.+2 \alpha_{n}\left\langle\varphi\left(x_{n}\right)-p, t_{n}-p\right\rangle\right) \\
= & \left(1-\Delta_{n}\right)\left\|z_{n}-p\right\|^{2}+2 \Delta_{n}\left\langle\varphi\left(x_{n}\right)-p, t_{n}-p\right\rangle-\beta_{n}\left(1-\beta_{n}\right)\left\|S t_{n}-z_{n}\right\|^{2},
\end{aligned}
$$

where $0<\Delta_{n}=\alpha_{n}\left(1-\beta_{n}\right)<1$. Combining Lemma 3.2, (3.19), and (3.20), we obtain

$$
\begin{aligned}
& \delta_{n}^{*}\left(\left\|y_{n}-w_{n}\right\|^{2}+\left\|y_{n}-z_{n}\right\|^{2}\right)+\beta_{n}\left(1-\beta_{n}\right)\left\|S t_{n}-z_{n}\right\|^{2} \\
\leq & \left\|w_{n}-p\right\|^{2}-\left\|x_{n+1}-p\right\|^{2}+2 \Delta_{n}\left\langle\varphi\left(x_{n}\right)-p, t_{n}-p\right\rangle \\
\leq & \left\|x_{n}-p\right\|^{2}-\left\|x_{n+1}-p\right\|^{2}+2 \Delta_{n}\left\langle\varphi\left(x_{n}\right)-p, t_{n}-p\right\rangle+\alpha_{n} M_{2} .
\end{aligned}
$$

\section{Claim 3.}

$$
\begin{aligned}
\left\|x_{n+1}-p\right\|^{2} \leq & \left(1-(1-\rho) \alpha_{n}\left(1-\beta_{n}\right)\right)\left\|x_{n}-p\right\|^{2}+(1-\rho) \alpha_{n}\left(1-\beta_{n}\right) \times \\
& {\left[\frac{2}{1-\rho}\left\langle\varphi(p)-p, t_{n}-p\right\rangle+\frac{3 M \theta_{n}}{(1-\rho) \alpha_{n}\left(1-\beta_{n}\right)}\left\|x_{n}-x_{n-1}\right\|\right], \forall n>n_{0} }
\end{aligned}
$$

for some $M>0$. Indeed, by the definition of $w_{n}$, one obtains

$$
\begin{aligned}
\left\|w_{n}-p\right\|^{2} & =\left\|x_{n}-p\right\|^{2}+2 \theta_{n}\left\langle x_{n}-p, x_{n}-x_{n-1}\right\rangle+\theta_{n}^{2}\left\|x_{n}-x_{n-1}\right\|^{2} \\
& \leq\left\|x_{n}-p\right\|^{2}+3 M \theta_{n}\left\|x_{n}-x_{n-1}\right\|,
\end{aligned}
$$

where $M:=\sup _{n \in \mathbb{N}}\left\{\left\|x_{n}-p\right\|, \theta\left\|x_{n}-x_{n-1}\right\|\right\}>0$. From (2.1), (2.2), (3.18), (3.20), and (3.21), for all $n \geq n_{0}$, one has

$$
\begin{aligned}
& \left\|x_{n+1}-p\right\|^{2} \leq \beta_{n}\left\|z_{n}-p\right\|^{2}+\left(1-\beta_{n}\right)\left\|S t_{n}-p\right\|^{2} \\
\leq & \beta_{n}\left\|z_{n}-p\right\|^{2}+\left(1-\beta_{n}\right) \times\left\|\alpha_{n}\left(\varphi\left(x_{n}\right)-\varphi(p)\right)+\left(1-\alpha_{n}\right)\left(z_{n}-p\right)+\alpha_{n}(\varphi(p)-p)\right\|^{2} \\
\leq & \beta_{n}\left\|z_{n}-p\right\|^{2}+2\left(1-\beta_{n}\right) \alpha_{n}\left\langle\varphi(p)-p, t_{n}-p\right\rangle \\
& +\left(1-\beta_{n}\right)\left\|\alpha_{n}\left(\varphi\left(x_{n}\right)-\varphi(p)\right)+\left(1-\alpha_{n}\right)\left(z_{n}-p\right)\right\|^{2} \\
\leq & \beta_{n}\left\|z_{n}-p\right\|^{2}+2\left(1-\beta_{n}\right) \alpha_{n}\left\langle\varphi(p)-p, t_{n}-p\right\rangle \\
& +\left(1-\beta_{n}\right) \alpha_{n}\left\|\left(\varphi\left(x_{n}\right)-\varphi(p)\right)\right\|^{2}+\left(1-\beta_{n}\right)\left(1-\alpha_{n}\right)\left\|\left(z_{n}-p\right)\right\|^{2} \\
= & \left(1-\Delta_{n}\right)\left\|z_{n}-p\right\|^{2}+\rho \Delta_{n}\left\|x_{n}-p\right\|^{2}+2 \Delta_{n}\left\langle\varphi(p)-p, t_{n}-p\right\rangle \\
\leq & \left(1-(1-\rho) \Delta_{n}\right)\left\|x_{n}-p\right\|^{2}+(1-\rho) \Delta_{n}\left(\frac{2}{1-\rho}\left\langle\varphi(p)-p, t_{n}-p\right\rangle+\frac{3 M \theta_{n}}{(1-\rho) \Delta_{n}}\left\|x_{n}-x_{n-1}\right\|\right),
\end{aligned}
$$

where $0<\Delta_{n}=\alpha_{n}\left(1-\beta_{n}\right)<1$.

Claim 4. The sequence $\left\{\left\|x_{n}-p\right\|^{2}\right\}$ converges to zero by considering two possible cases on $\left\{\left\|x_{n}-p\right\|^{2}\right\}$. 
Case 1. There exists $N \in \mathbb{N}$ such that $\left\|x_{n+1}-p\right\|^{2} \leq\left\|x_{n}-p\right\|^{2}$ for all $n \geq N$. This together with the boundedness of $\left\{\left\|x_{n}-p\right\|^{2}\right\}$ yields that $\lim _{n \rightarrow \infty}\left\|x_{n}-p\right\|^{2}$ exists. From Claim 2 , Conditions (C5) and (C6), and $\lim _{n \rightarrow \infty} \delta_{n}^{*}>0$, we obtain

$$
\lim _{n \rightarrow \infty}\left\|S t_{n}-z_{n}\right\|=0, \lim _{n \rightarrow \infty}\left\|w_{n}-y_{n}\right\|=0, \lim _{n \rightarrow \infty}\left\|z_{n}-y_{n}\right\|=0,
$$

which means that $\lim _{n \rightarrow \infty}\left\|z_{n}-w_{n}\right\|=0$. According to the definition of $w_{n}$ and Remark 3.2 (i), one has

$$
\left\|x_{n}-w_{n}\right\|=\alpha_{n} \cdot \frac{\theta_{n}}{\alpha_{n}}\left\|x_{n}-x_{n-1}\right\| \rightarrow 0 \text { as } n \rightarrow \infty .
$$

This together with $\lim _{n \rightarrow \infty}\left\|z_{n}-w_{n}\right\|=0$ implies that

$$
\lim _{n \rightarrow \infty}\left\|z_{n}-x_{n}\right\|=0 \text {. }
$$

As $\left\{x_{n}\right\}$ is bounded, there exists a subsequence $\left\{x_{n_{k}}\right\}$ of $\left\{x_{n}\right\}$ that converges weakly to some $z \in \mathscr{H}$. Moreover,

$$
\limsup _{n \rightarrow \infty}\left\langle\varphi(p)-p, x_{n}-p\right\rangle=\lim _{k \rightarrow \infty}\left\langle\varphi(p)-p, x_{n_{k}}-p\right\rangle=\langle\varphi(p)-p, z-p\rangle .
$$

It follows from (3.22) and (3.23) that $y_{n_{k}} \rightarrow z$ and $z \in C$. By means of $y_{n_{k}}=\operatorname{prox}_{\lambda_{n_{k}} f\left(w_{n_{k}}, \cdot\right)}\left(w_{n_{k}}\right)$ and Lemma 2.2, we obtain

$$
\lambda_{n_{k}}\left(f\left(w_{n_{k}}, y\right)-f\left(w_{n_{k}}, y_{n_{k}}\right)\right) \geq\left\langle w_{n_{k}}-y_{n_{k}}, y-y_{n_{k}}\right\rangle, \quad \forall y \in C .
$$

Passing to the limit in (3.26) as $k \rightarrow \infty$ and using the Assumptions (A1), (A4), and Lemma 3.1, we obtain $f(z, y) \geq 0, \forall y \in C$, which implies that $z \in \mathrm{EP}(f)$. Next we show that $z \in \mathrm{F}(S)$. From $t_{n}-z_{n}=\alpha_{n}\left(\varphi\left(x_{n}\right)-z_{n}\right)$ and Condition (C5), one has $\lim _{n \rightarrow \infty}\left\|t_{n}-z_{n}\right\|=0$. Thus, we deduce from (3.22) that $t_{n_{k}} \rightarrow z$ and $\lim _{n \rightarrow \infty}\left\|t_{n}-S t_{n}\right\|=0$. It follows from the demiclosedness of $(I-S)$ that $z \in \mathrm{F}(S)$. Consequently, we conclude that $z \in \mathrm{EP}(f) \cap \mathrm{F}(S)$. Combining (3.14) and (3.25), we obtain

$$
\limsup _{n \rightarrow \infty}\left\langle\varphi(p)-p, x_{n}-p\right\rangle=\langle\varphi(p)-p, z-p\rangle \leq 0 .
$$

It follows from (3.24) and $\lim _{n \rightarrow \infty}\left\|t_{n}-z_{n}\right\|=0$ that $\lim _{n \rightarrow \infty}\left\|t_{n}-x_{n}\right\|=0$, which together with (3.27) yields

$$
\begin{aligned}
& \limsup _{n \rightarrow \infty}\left\langle\varphi(p)-p, t_{n}-p\right\rangle \\
\leq & \limsup _{n \rightarrow \infty}\left\langle\varphi(p)-p, t_{n}-x_{n}\right\rangle+\limsup _{n \rightarrow \infty}\left\langle\varphi(p)-p, x_{n}-p\right\rangle \leq 0 .
\end{aligned}
$$

Combining Claim 3, (3.28), Conditions (C5), (C6), and Remark 3.2 (i), we conclude from Lemma 2.4 that $\lim _{n \rightarrow \infty}\left\|x_{n}-p\right\|=0$.

Case 2. There exists a subsequence $\left\{\left\|x_{n_{j}}-p\right\|\right\}$ of $\left\{\left\|x_{n}-p\right\|\right\}$ such that $\left\|x_{n_{j}}-p\right\|<\| x_{n_{j}+1}-$ $p \|$ for all $j \in \mathbb{N}$. From Lemma 2.3, there exists a nondecreasing sequence $m_{k}$ of $\mathbb{N}$ such that $\lim _{k \rightarrow \infty} m_{k}=\infty$ and the following inequalities hold for all $k \in \mathbb{N}$ :

$$
\left\|x_{m_{k}}-p\right\| \leq\left\|x_{m_{k}+1}-p\right\| \text { and }\left\|x_{k}-p\right\| \leq\left\|x_{m_{k}+1}-p\right\| \text {. }
$$

It follows from Remark 3.3 that $\lim _{k \rightarrow \infty} \delta_{m_{k}}^{*}>0$. According to Claim 2 and (3.29), we have

$$
\begin{aligned}
& \delta_{m_{k}}^{*}\left(\left\|y_{m_{k}}-w_{m_{k}}\right\|^{2}+\left\|y_{m_{k}}-z_{m_{k}}\right\|^{2}\right)+\beta_{m_{k}}\left(1-\beta_{m_{k}}\right)\left\|S t_{m_{k}}-z_{m_{k}}\right\|^{2} \\
\leq & \left\|x_{m_{k}}-p\right\|^{2}-\left\|x_{m_{k}+1}-p\right\|^{2}+2 \alpha_{m_{k}}\left(1-\beta_{m_{k}}\right)\left\langle\varphi\left(x_{m_{k}}\right)-p, t_{m_{k}}-p\right\rangle+\alpha_{m_{k}} M_{2} \\
\leq & 2 \alpha_{m_{k}}\left(1-\beta_{m_{k}}\right)\left\langle\varphi\left(x_{m_{k}}\right)-p, t_{m_{k}}-p\right\rangle+\alpha_{m_{k}} M_{2} .
\end{aligned}
$$


This combining with $\lim _{k \rightarrow \infty} \delta_{m_{k}}^{*}>0$, Conditions (C5) and (C6) yield that

$$
\lim _{k \rightarrow \infty}\left\|w_{m_{k}}-y_{m_{k}}\right\|=0, \lim _{k \rightarrow \infty}\left\|z_{m_{k}}-y_{m_{k}}\right\|=0, \lim _{k \rightarrow \infty}\left\|S t_{m_{k}}-z_{m_{k}}\right\|=0 .
$$

Using the same arguments (3.23)-(3.28) as in the proof of Case 1, we obtain

$$
\limsup _{k \rightarrow \infty}\left\langle\varphi(p)-p, t_{m_{k}}-p\right\rangle \leq 0 .
$$

Combining Claim 3 and (3.29), we have for all $m_{k} \geq n_{0}$,

$$
\begin{aligned}
\left\|x_{m_{k}+1}-p\right\|^{2} \leq & \left(1-(1-\rho) \alpha_{m_{k}}\left(1-\beta_{m_{k}}\right)\right)\left\|x_{m_{k}+1}-p\right\|^{2}+(1-\rho) \alpha_{m_{k}}\left(1-\beta_{m_{k}}\right) \times \\
& \left(\frac{2}{1-\rho}\left\langle\varphi(p)-p, t_{m_{k}}-p\right\rangle+\frac{3 M \theta_{m_{k}}}{(1-\rho) \alpha_{m_{k}}\left(1-\beta_{m_{k}}\right)}\left\|x_{m_{k}}-x_{m_{k}-1}\right\|\right),
\end{aligned}
$$

which further yields that

$$
\left\|x_{m_{k}+1}-p\right\|^{2} \leq \frac{2}{1-\rho}\left\langle\varphi(p)-p, t_{m_{k}}-p\right\rangle+\frac{3 M \theta_{m_{k}}}{(1-\rho) \alpha_{m_{k}}\left(1-\beta_{m_{k}}\right)}\left\|x_{m_{k}}-x_{m_{k}-1}\right\|, \forall m_{k} \geq n_{0} .
$$

This together with (3.30) and Remark 3.2 (i) implies that $\lim _{k \rightarrow \infty}\left\|x_{m_{k}+1}-p\right\|=0$. It follows from (3.29) that $\lim _{k \rightarrow \infty}\left\|x_{k}-p\right\|=0$, i.e., $x_{k} \rightarrow p$ as $k \rightarrow \infty$. This completes the proof.

3.2. The Algorithm 3.2 and its convergence analysis. In this subsection, we present a new iterative scheme that combines the inertial subgradient extragradient method and the viscositylike method for finding a common solution to the pseudomonotone equilibrium problem and the fixed point problem with a demicontractive mapping. We assume that the proposed Algorithm 3.2 satisfies Conditions (C1), (C2), (C4), (C5) and the following two conditions.

(C7) The mapping $S: \mathscr{H} \rightarrow \mathscr{H}$ is $\lambda$-demicontractive such that $(I-S)$ is demiclosed at zero.

(C8) Let $\left\{\beta_{n}\right\}$ be a real sequence in $(0,1)$ such that $\left\{\beta_{n}\right\} \subset(a, 1-\lambda)$ for some $a>0$.

The proposed Algorithm 3.2 is stated as follows.

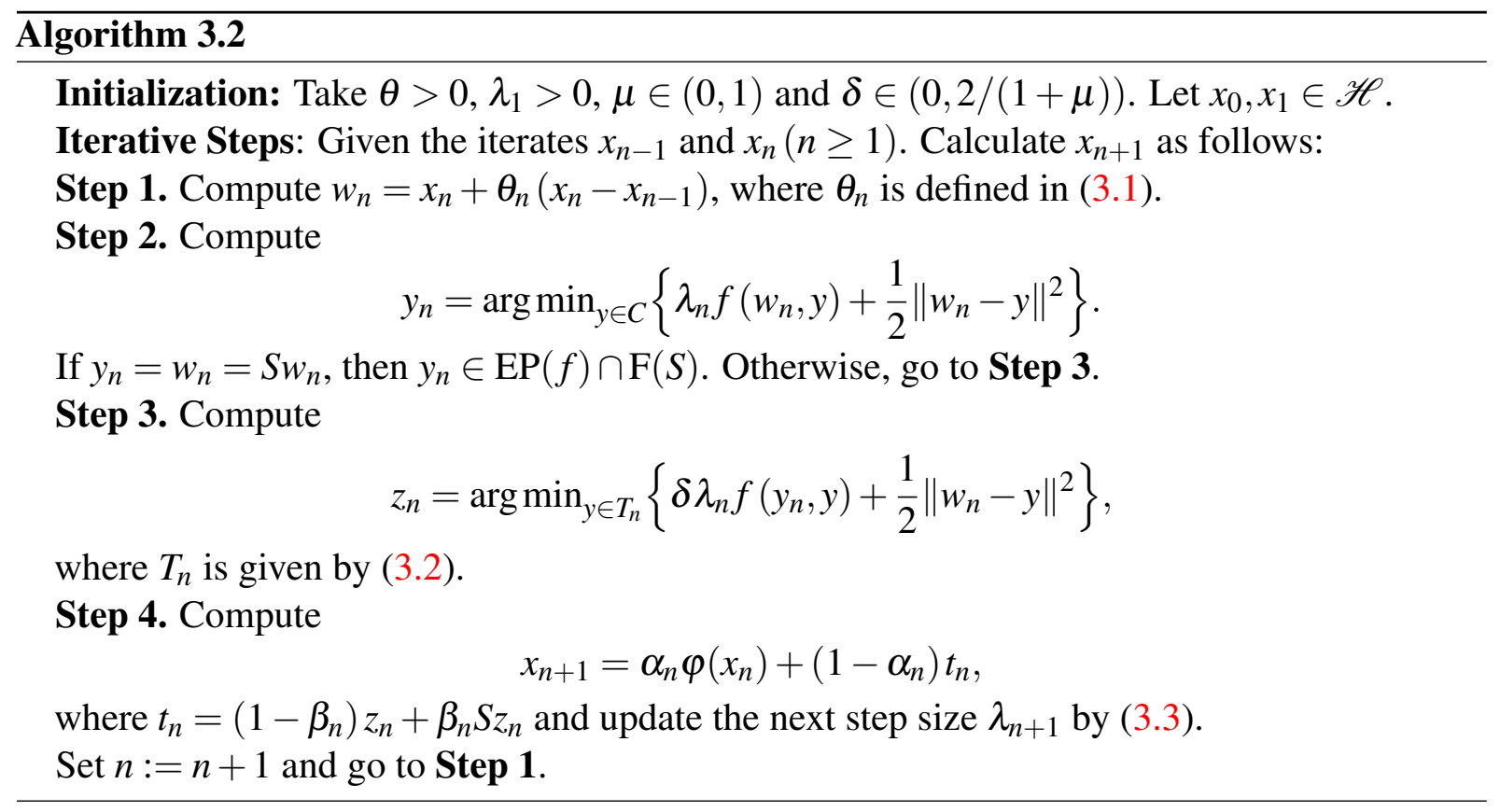


Theorem 3.2. Assume that Conditions (C1), (C2), (C4), (C5), (C7), and (C8) hold. Then the sequence $\left\{x_{n}\right\}$ generated by Algorithm 3.2 converges to $p \in \operatorname{EP}(f) \cap \mathrm{F}(S)$ in norm, where $p=P_{\mathrm{EP}(f) \cap \mathrm{F}(S)}(\varphi(p))$.

Proof. For convenience, we divide the proof into four claims.

Claim 1. The sequence $\left\{x_{n}\right\}$ is bounded. From the definition of $t_{n}$ and Lemma 2.5 (ii), we have

$$
\left\|t_{n}-p\right\|^{2} \leq\left\|z_{n}-p\right\|^{2}-\beta_{n}\left(1-\lambda-\beta_{n}\right)\left\|S z_{n}-z_{n}\right\|^{2} .
$$

Using (3.31) and Lemma 3.2, we have

$$
\begin{aligned}
\left\|t_{n}-p\right\|^{2} \leq & \left\|w_{n}-p\right\|^{2}-\delta_{n}^{*}\left(\left\|w_{n}-y_{n}\right\|^{2}+\left\|z_{n}-y_{n}\right\|^{2}\right) \\
& -\beta_{n}\left(1-\lambda-\beta_{n}\right)\left\|S z_{n}-z_{n}\right\|^{2} .
\end{aligned}
$$

It follows from Remark 3.3 and $\left\{\beta_{n}\right\} \subset(a, 1-\lambda)$ that

$$
\left\|t_{n}-p\right\| \leq\left\|w_{n}-p\right\|, \quad \forall n \geq n_{0} .
$$

Combining (3.16), (3.17), and (3.33), one obtains

$$
\left\|t_{n}-p\right\| \leq\left\|w_{n}-p\right\| \leq\left\|x_{n}-p\right\|+\alpha_{n} M_{1}, \quad \forall n \geq n_{0} .
$$

From the definition of $x_{n+1}$ and (3.34), we have

$$
\begin{aligned}
\left\|x_{n+1}-p\right\| & =\left\|\alpha_{n} \varphi\left(x_{n}\right)+\left(1-\alpha_{n}\right) t_{n}-p\right\| \\
& \leq \alpha_{n}\left\|\varphi\left(x_{n}\right)-\varphi(p)\right\|+\alpha_{n}\|\varphi(p)-p\|+\left(1-\alpha_{n}\right)\left\|t_{n}-p\right\| \\
& \leq \alpha_{n} \rho\left\|x_{n}-p\right\|+\alpha_{n}\|\varphi(p)-p\|+\left(1-\alpha_{n}\right)\left\|w_{n}-p\right\| \\
& \leq\left[1-\alpha_{n}(1-\rho)\right]\left\|x_{n}-p\right\|+\alpha_{n}(1-\rho) \frac{\|\varphi(p)-p\|+M_{1}}{1-\rho} \\
& \leq \max \left\{\left\|x_{n}-p\right\|, \frac{\|\varphi(p)-p\|+M_{1}}{1-\rho}\right\} \\
& \leq \cdots \leq \max \left\{\left\|x_{n_{0}}-p\right\|, \frac{\|\varphi(p)-p\|+M_{1}}{1-\rho}\right\}, \forall n \geq n_{0} .
\end{aligned}
$$

That is, $\left\{x_{n}\right\}$ is bounded, so are $\left\{\varphi\left(x_{n}\right)\right\},\left\{w_{n}\right\},\left\{y_{n}\right\},\left\{z_{n}\right\}$, and $\left\{t_{n}\right\}$.

\section{Claim 2.}

$$
\begin{aligned}
& \left(1-\alpha_{n}\right) \delta_{n}^{*}\left(\left\|y_{n}-w_{n}\right\|^{2}+\left\|z_{n}-y_{n}\right\|^{2}\right)+\left(1-\alpha_{n}\right) \beta_{n}\left(1-\lambda-\beta_{n}\right)\left\|S z_{n}-z_{n}\right\|^{2} \\
\leq & \left\|x_{n}-p\right\|^{2}-\left\|x_{n+1}-p\right\|^{2}+\alpha_{n}\left\|\varphi\left(x_{n}\right)-p\right\|^{2}+\alpha_{n} M_{2}
\end{aligned}
$$

for some $M_{2}>0$. Using (2.2), (3.19), and (3.32), we have

$$
\begin{aligned}
\left\|x_{n+1}-p\right\|^{2}= & \left\|\alpha_{n}\left(\varphi\left(x_{n}\right)-p\right)+\left(1-\alpha_{n}\right)\left(t_{n}-p\right)\right\|^{2} \\
\leq & \alpha_{n}\left\|\varphi\left(x_{n}\right)-p\right\|^{2}+\left(1-\alpha_{n}\right)\left\|t_{n}-p\right\|^{2} \\
\leq & \alpha_{n}\left\|\varphi\left(x_{n}\right)-p\right\|^{2}+\left\|x_{n}-p\right\|^{2}+\alpha_{n} M_{2}-\left(1-\alpha_{n}\right) \delta_{n}^{*}\left(\left\|y_{n}-w_{n}\right\|^{2}+\left\|z_{n}-y_{n}\right\|^{2}\right) \\
& -\left(1-\alpha_{n}\right) \beta_{n}\left(1-\lambda-\beta_{n}\right)\left\|S z_{n}-z_{n}\right\|^{2} .
\end{aligned}
$$

The desired result now follows easily. 
Claim 3.

$$
\begin{aligned}
\left\|x_{n+1}-p\right\|^{2} \leq & \left(1-(1-\rho) \alpha_{n}\right)\left\|x_{n}-p\right\|^{2}+(1-\rho) \alpha_{n}\left[\frac{2}{1-\rho}\left\langle\varphi(p)-p, x_{n+1}-p\right\rangle\right. \\
& \left.+\frac{3 M \theta_{n}}{(1-\rho) \alpha_{n}}\left\|x_{n}-x_{n-1}\right\|\right], \quad \forall n \geq n_{0}
\end{aligned}
$$

for some $M>0$. Combining (2.1), (2.2), (3.21), and (3.33), we obtain

$$
\begin{aligned}
\left\|x_{n+1}-p\right\|^{2} & =\left\|\alpha_{n} \varphi\left(x_{n}\right)+\left(1-\alpha_{n}\right) t_{n}-p\right\|^{2} \\
& =\left\|\alpha_{n}\left(\varphi\left(x_{n}\right)-\varphi(p)\right)+\left(1-\alpha_{n}\right)\left(t_{n}-p\right)+\alpha_{n}(\varphi(p)-p)\right\|^{2} \\
\leq & \left\|\alpha_{n}\left(\varphi\left(x_{n}\right)-\varphi(p)\right)+\left(1-\alpha_{n}\right)\left(t_{n}-p\right)\right\|^{2}+2 \alpha_{n}\left\langle\varphi(p)-p, x_{n+1}-p\right\rangle \\
\leq & \alpha_{n}\left\|\varphi\left(x_{n}\right)-\varphi(p)\right\|^{2}+\left(1-\alpha_{n}\right)\left\|t_{n}-p\right\|^{2}+2 \alpha_{n}\left\langle\varphi(p)-p, x_{n+1}-p\right\rangle \\
\leq & \alpha_{n} \rho\left\|x_{n}-p\right\|^{2}+\left(1-\alpha_{n}\right)\left\|x_{n}-p\right\|^{2}+2 \alpha_{n}\left\langle\varphi(p)-p, x_{n+1}-p\right\rangle+3 M \theta_{n}\left\|x_{n}-x_{n-1}\right\| \\
= & \left(1-(1-\rho) \alpha_{n}\right)\left\|x_{n}-p\right\|^{2}+(1-\rho) \alpha_{n}\left[\frac{2}{1-\rho}\left\langle\varphi(p)-p, x_{n+1}-p\right\rangle\right. \\
& \left.+\frac{3 M \theta_{n}}{(1-\rho) \alpha_{n}}\left\|x_{n}-x_{n-1}\right\|\right], \quad \forall n \geq n_{0} .
\end{aligned}
$$

Claim 4. The sequence $\left\{\left\|x_{n}-p\right\|^{2}\right\}$ converges to zero by considering two possible cases on $\left\{\left\|x_{n}-p\right\|^{2}\right\}$.

Case 1. There exists an $N \in \mathbb{N}$ such that $\left\|x_{n+1}-p\right\|^{2} \leq\left\|x_{n}-p\right\|^{2}$ for all $n \geq N$. This implies that $\lim _{n \rightarrow \infty}\left\|x_{n}-p\right\|^{2}$ exists. By Claim 2, Conditions (C5), (C8), and $\lim _{n \rightarrow \infty} \delta_{n}^{*}>0$, we obtain

$$
\lim _{n \rightarrow \infty}\left\|w_{n}-y_{n}\right\|=0, \lim _{n \rightarrow \infty}\left\|z_{n}-y_{n}\right\|=0, \lim _{n \rightarrow \infty}\left\|z_{n}-S z_{n}\right\|=0,
$$

which implies that $\lim _{n \rightarrow \infty}\left\|z_{n}-w_{n}\right\|=0$. From (3.23) and (3.24), we obtain

$$
\lim _{n \rightarrow \infty}\left\|x_{n}-w_{n}\right\|=0 \text { and } \lim _{n \rightarrow \infty}\left\|z_{n}-x_{n}\right\|=0 .
$$

By the definition of $t_{n}$, i.e., $t_{n}=\left(1-\beta_{n}\right) z_{n}+\beta_{n} S z_{n}$, one sees that

$$
\left\|t_{n}-z_{n}\right\|=\beta_{n}\left\|S z_{n}-z_{n}\right\| \leq(1-\lambda)\left\|S z_{n}-z_{n}\right\|
$$

In view of (3.35), we obtain

$$
\lim _{n \rightarrow \infty}\left\|t_{n}-z_{n}\right\|=0
$$

From the hypothesis on $\left\{\alpha_{n}\right\},(3.36)$, and (3.37), we deduce

$$
\begin{aligned}
\left\|x_{n+1}-x_{n}\right\| & =\left\|\alpha_{n} \varphi\left(x_{n}\right)+\left(1-\alpha_{n}\right) t_{n}-x_{n}\right\| \\
& \leq \alpha_{n}\left\|\varphi\left(x_{n}\right)-x_{n}\right\|+\left(1-\alpha_{n}\right)\left\|t_{n}-x_{n}\right\| \\
& \leq \alpha_{n}\left\|\varphi\left(x_{n}\right)-x_{n}\right\|+\left\|t_{n}-z_{n}\right\|+\left\|z_{n}-x_{n}\right\| \rightarrow 0 \text { as } n \rightarrow \infty .
\end{aligned}
$$

With the help of the same facts as in (3.25) and (3.26), we can also obtain that $z \in \operatorname{EP}(f)$. It follows from (3.36) that $z_{n_{k}} \rightarrow z$, which together with $\left\|z_{n}-S z_{n}\right\| \rightarrow 0$ and the demiclosedness of $(I-S)$ yields that $z \in \mathrm{F}(S)$. Thus, $z \in \mathrm{EP}(f) \cap \mathrm{F}(S)$. Combining the definition of $p, z \in$ $\mathrm{EP}(f) \cap \mathrm{F}(S)$, and (3.14), we obtain

$$
\limsup _{n \rightarrow \infty}\left\langle\varphi(p)-p, x_{n}-p\right\rangle=\langle\varphi(p)-p, z-p\rangle \leq 0 .
$$


This combining with (3.38) infers that

$$
\begin{aligned}
& \limsup _{n \rightarrow \infty}\left\langle\varphi(p)-p, x_{n+1}-p\right\rangle \\
\leq & \limsup _{n \rightarrow \infty}\left\langle\varphi(p)-p, x_{n+1}-x_{n}\right\rangle+\limsup _{n \rightarrow \infty}\left\langle\varphi(p)-p, x_{n}-p\right\rangle \\
= & \langle\varphi(p)-p, z-p\rangle \leq 0 .
\end{aligned}
$$

Using Claim 3, (3.39), Remark 3.2 (i), and Lemma 2.4, we observe that $\lim _{n \rightarrow \infty}\left\|x_{n}-p\right\|=0$. That is, $x_{n} \rightarrow p$ as $n \rightarrow \infty$.

Case 2. There exists a subsequence $\left\{\left\|x_{n_{j}}-p\right\|\right\}$ of $\left\{\left\|x_{n}-p\right\|\right\}$ such that $\left\|x_{n_{j}}-p\right\|<\| x_{n_{j}+1}-$ $p \|$ for all $j \in \mathbb{N}$. Combining Claim 2 and (3.29), we have

$$
\begin{aligned}
& \left(1-\alpha_{m_{k}}\right) \delta_{m_{k}}^{*}\left(\left\|y_{m_{k}}-w_{m_{k}}\right\|^{2}+\left\|z_{m_{k}}-y_{m_{k}}\right\|^{2}\right)+\left(1-\alpha_{m_{k}}\right) \beta_{m_{k}}\left(1-\lambda-\beta_{m_{k}}\right)\left\|S z_{m_{k}}-z_{m_{k}}\right\|^{2} \\
\leq & \left\|x_{m_{k}}-p\right\|^{2}-\left\|x_{m_{k}+1}-p\right\|^{2}+\alpha_{m_{k}}\left\|\varphi\left(x_{m_{k}}\right)-p\right\|^{2}+\alpha_{m_{k}} M_{2} \\
\leq & \alpha_{m_{k}}\left\|\varphi\left(x_{m_{k}}\right)-p\right\|^{2}+\alpha_{m_{k}} M_{2} .
\end{aligned}
$$

By the hypothesis on $\left\{\alpha_{n}\right\},\left\{\beta_{n}\right\}$, and Remark 3.3, we obtain

$$
\lim _{k \rightarrow \infty}\left\|w_{m_{k}}-y_{m_{k}}\right\|=0, \lim _{k \rightarrow \infty}\left\|z_{m_{k}}-y_{m_{k}}\right\|=0, \lim _{k \rightarrow \infty}\left\|z_{m_{k}}-S z_{m_{k}}\right\|=0 .
$$

Using the same arguments as in the proof of Case 1, we obtain

$$
\limsup _{k \rightarrow \infty}\left\langle\varphi(p)-p, x_{m_{k}+1}-p\right\rangle \leq 0 .
$$

Combining Claim 3 and (3.29), we deduce that

$$
\begin{aligned}
\left\|x_{m_{k}+1}-p\right\|^{2} \leq & \left(1-(1-\rho) \alpha_{m_{k}}\right)\left\|x_{m_{k}+1}-p\right\|^{2}+(1-\rho) \alpha_{m_{k}}\left[\frac{2}{1-\rho}\left\langle\varphi(p)-p, x_{m_{k}+1}-p\right\rangle\right. \\
& \left.+\frac{3 M \theta_{m_{k}}}{(1-\rho) \alpha_{m_{k}}}\left\|x_{m_{k}}-x_{m_{k}-1}\right\|\right], \quad \forall m_{k} \geq n_{0} .
\end{aligned}
$$

This follows that

$$
\left\|x_{m_{k}+1}-p\right\|^{2} \leq \frac{2}{1-\rho}\left\langle\varphi(p)-p, x_{m_{k}+1}-p\right\rangle+\frac{3 M \theta_{m_{k}}}{(1-\rho) \alpha_{m_{k}}}\left\|x_{m_{k}}-x_{m_{k}-1}\right\| .
$$

Combining Remark 3.2 (i), (3.29), (3.40), and (3.41), we obtain that $\limsup _{k \rightarrow \infty}\left\|x_{k}-p\right\|^{2} \leq 0$. This means that $x_{k} \rightarrow p$ as $k \rightarrow \infty$. The proof of Theorem 3.2 is completed.

3.3. The Algorithm 3.3 and its convergence analysis. In this subsection, we introduce a Mann-type subgradient extragradient method (see Algorithm 3.3 below) for solving the pseudomonotone equilibrium problem and the fixed point problem with a demicontractive mapping. It is assumed that Algorithm 3.3 satisfies Conditions (C1), (C2), (C5), (C7) and the following condition.

(C9) Let $\left\{\beta_{n}\right\}$ be a real sequence such that $\left\{\beta_{n}\right\} \subset(a, b) \subset\left(a,(1-\lambda)\left(1-\alpha_{n}\right)\right)$ for some $b>a>0$.

The proposed Algorithm 3.3 is illustrated below.

Theorem 3.3. Assume that Conditions (C1), (C2), (C5), (C7), and (C9) hold. Then the sequence $\left\{x_{n}\right\}$ generated by Algorithm 3.3 converges to $p \in \operatorname{EP}(f) \cap \mathrm{F}(S)$ in norm, where $\|p\|=\min \{\|z\|$ : $z \in \mathrm{EP}(f) \cap \mathrm{F}(S)\}$. 


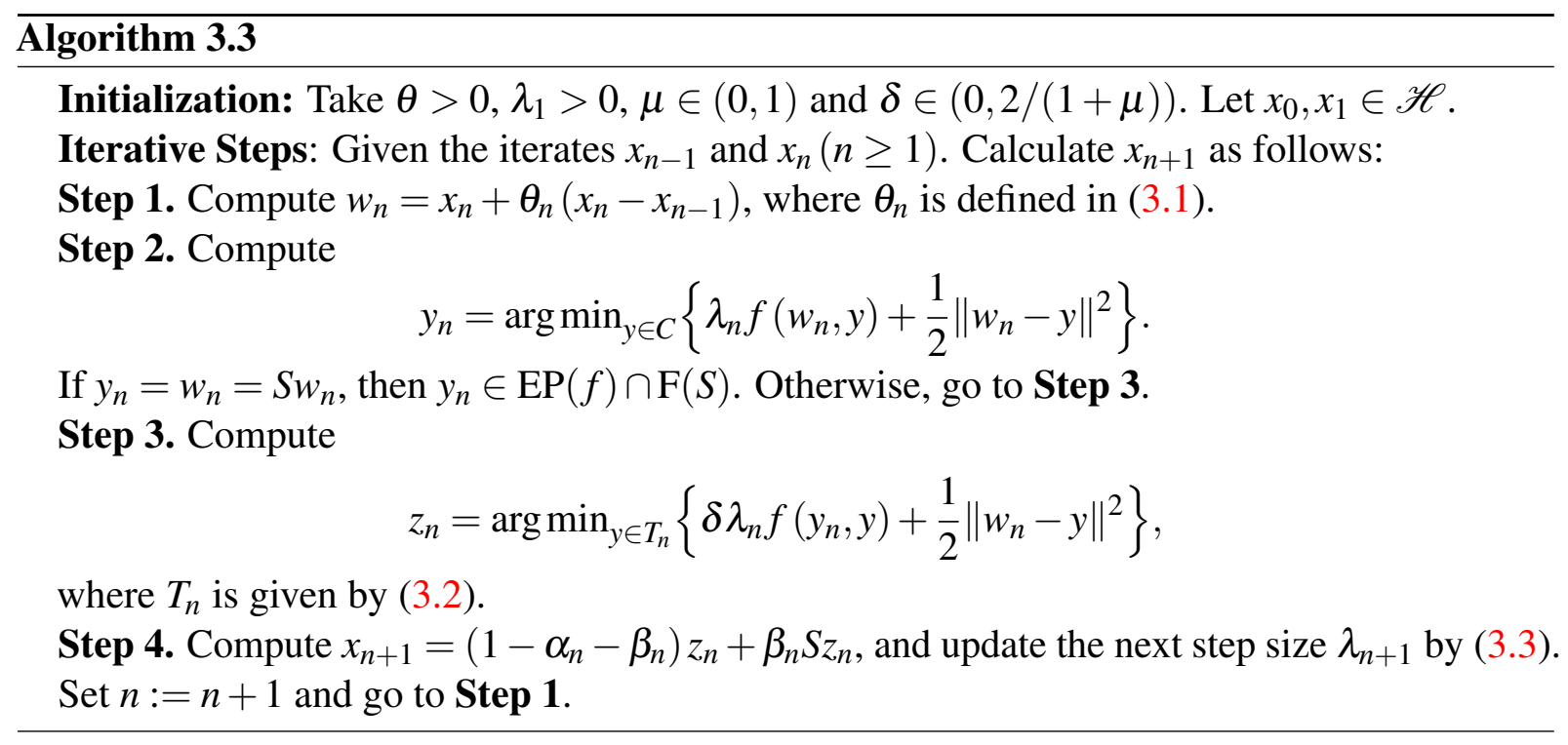

Proof. We also divided the proof into four parts.

Claim 1. The sequence $\left\{x_{n}\right\}$ is bounded. From the definiton of $x_{n+1}$, one has

$$
\left\|x_{n+1}-p\right\| \leq\left\|\left(1-\alpha_{n}-\beta_{n}\right)\left(z_{n}-p\right)+\beta_{n}\left(S z_{n}-p\right)\right\|+\alpha_{n}\|p\| .
$$

Combining (2.4), (2.6), (3.15), and the hypothesis on $\left\{\beta_{n}\right\}$, we have

$$
\begin{aligned}
& \left\|\left(1-\alpha_{n}-\beta_{n}\right)\left(z_{n}-p\right)+\beta_{n}\left(S z_{n}-p\right)\right\|^{2} \\
= & \left(1-\alpha_{n}-\beta_{n}\right)^{2}\left\|z_{n}-p\right\|^{2}+\beta_{n}^{2}\left\|S z_{n}-p\right\|^{2}+2\left(1-\alpha_{n}-\beta_{n}\right) \beta_{n}\left\langle S z_{n}-p, z_{n}-p\right\rangle \\
\leq & \left(1-\alpha_{n}-\beta_{n}\right)^{2}\left\|z_{n}-p\right\|^{2}+\beta_{n}^{2}\left[\left\|z_{n}-p\right\|^{2}+\lambda\left\|z_{n}-S z_{n}\right\|^{2}\right] \\
& +2\left(1-\alpha_{n}-\beta_{n}\right) \beta_{n}\left[\left\|z_{n}-p\right\|^{2}-\frac{1-\lambda}{2}\left\|z_{n}-S z_{n}\right\|^{2}\right] \\
= & \left(1-\alpha_{n}\right)^{2}\left\|z_{n}-p\right\|^{2}+\beta_{n}\left(\beta_{n}-(1-\lambda)\left(1-\alpha_{n}\right)\right)\left\|z_{n}-S z_{n}\right\|^{2} \\
\leq & \left(1-\alpha_{n}\right)^{2}\left\|w_{n}-p\right\|^{2},
\end{aligned}
$$

which implies that

$$
\left\|\left(1-\alpha_{n}-\beta_{n}\right)\left(z_{n}-p\right)+\beta_{n}\left(S z_{n}-p\right)\right\| \leq\left(1-\alpha_{n}\right)\left\|w_{n}-p\right\|, \quad \forall n \geq n_{0} .
$$

From (3.15), (3.16), and (3.17), we find that

$$
\left\|z_{n}-p\right\| \leq\left\|w_{n}-p\right\| \leq\left\|x_{n}-p\right\|+\alpha_{n} M_{1}, \quad \forall n \geq n_{0} .
$$

Combining (3.42), (3.43), and (3.44), we obtain

$$
\begin{aligned}
\left\|x_{n+1}-p\right\| & \leq\left(1-\alpha_{n}\right)\left\|w_{n}-p\right\|+\alpha_{n}\|p\| \\
& \leq\left(1-\alpha_{n}\right)\left\|x_{n}-p\right\|+\alpha_{n}\left(\|p\|+M_{1}\right) \\
& \leq \max \left\{\left\|x_{n}-p\right\|,\|p\|+M_{1}\right\} \\
& \leq \cdots \leq \max \left\{\left\|x_{n_{0}}-p\right\|,\|p\|+M_{1}\right\} .
\end{aligned}
$$

Thus, the sequence $\left\{x_{n}\right\}$ is bounded, so are $\left\{w_{n}\right\},\left\{y_{n}\right\}$, and $\left\{z_{n}\right\}$. 


\section{Claim 2.}

$$
\begin{aligned}
& \beta_{n}\left[(1-\lambda)-\beta_{n}\right]\left\|z_{n}-S z_{n}\right\|^{2}+\delta_{n}^{*}\left(\left\|y_{n}-w_{n}\right\|^{2}+\left\|z_{n}-y_{n}\right\|^{2}\right) \\
\leq & \left\|x_{n}-p\right\|^{2}-\left\|x_{n+1}-p\right\|^{2}+\alpha_{n} M_{4}
\end{aligned}
$$

for some $M_{4}>0$. Combining (2.5), (3.19), (3.44), and Lemma 3.2, we have

$$
\begin{aligned}
\left\|x_{n+1}-p\right\|^{2} & =\left\|\left(z_{n}-p\right)+\beta_{n}\left(S z_{n}-z_{n}\right)-\alpha_{n} z_{n}\right\|^{2} \\
& \leq\left\|\left(z_{n}-p\right)+\beta_{n}\left(S z_{n}-z_{n}\right)\right\|^{2}-2 \alpha_{n}\left\langle z_{n}, x_{n+1}-p\right\rangle \\
& \leq\left\|z_{n}-p\right\|^{2}+\beta_{n}^{2}\left\|S z_{n}-z_{n}\right\|^{2}+\beta_{n}(\lambda-1)\left\|z_{n}-S z_{n}\right\|^{2}+\alpha_{n} M_{3} \\
& \leq\left\|x_{n}-p\right\|^{2}+\alpha_{n} M_{4}-\beta_{n}\left[(1-\lambda)-\beta_{n}\right]\left\|z_{n}-S z_{n}\right\|^{2}-\delta_{n}^{*}\left(\left\|y_{n}-w_{n}\right\|^{2}+\left\|z_{n}-y_{n}\right\|^{2}\right),
\end{aligned}
$$

where $M_{3}:=2 \sup _{n \in \mathbb{N}}\left|\left\langle z_{n}, p-x_{n+1}\right\rangle\right|$ and $M_{4}:=M_{2}+M_{3}$. Thus, we can obtain the desired result immediately.

\section{Claim 3.}

$$
\begin{aligned}
\left\|x_{n+1}-p\right\|^{2} \leq & \left(1-\alpha_{n}\right)\left\|x_{n}-p\right\|^{2}+\alpha_{n}\left[2 \beta_{n}\left\|z_{n}-S z_{n}\right\|\left\|x_{n+1}-p\right\|+2\left\langle p, p-x_{n+1}\right\rangle\right. \\
& \left.+\frac{3 M \theta_{n}}{\alpha_{n}}\left\|x_{n}-x_{n-1}\right\|\right], \quad \forall n \geq n_{0}
\end{aligned}
$$

for some $M>0$. Indeed, set $t_{n}=\left(1-\beta_{n}\right) z_{n}+\beta_{n} S z_{n}$. From the hypothesis on $\left\{\beta_{n}\right\},(3.31)$ and (3.44), one has

$$
\left\|t_{n}-p\right\| \leq\left\|z_{n}-p\right\| \leq\left\|w_{n}-p\right\|, \quad \forall n \geq n_{0} .
$$

In addition, one sees that

$$
\begin{aligned}
x_{n+1} & =t_{n}-\alpha_{n} z_{n}=\left(1-\alpha_{n}\right) t_{n}-\alpha_{n}\left(z_{n}-t_{n}\right) \\
& =\left(1-\alpha_{n}\right) t_{n}-\alpha_{n} \beta_{n}\left(z_{n}-S z_{n}\right) .
\end{aligned}
$$

From (2.1), (3.21), and (3.45), one has

$$
\begin{aligned}
\left\|x_{n+1}-p\right\|^{2}= & \left\|\left(1-\alpha_{n}\right)\left(t_{n}-p\right)-\alpha_{n}\left(\beta_{n}\left(z_{n}-S z_{n}\right)+p\right)\right\|^{2} \\
\leq & \left(1-\alpha_{n}\right)^{2}\left\|t_{n}-p\right\|^{2}-2 \alpha_{n}\left\langle\beta_{n}\left(z_{n}-S z_{n}\right)+p, x_{n+1}-p\right\rangle \\
= & \left(1-\alpha_{n}\right)^{2}\left\|t_{n}-p\right\|^{2}+\alpha_{n}\left[2 \beta_{n}\left\langle z_{n}-S z_{n}, p-x_{n+1}\right\rangle+2\left\langle p, p-x_{n+1}\right\rangle\right] \\
\leq & \left(1-\alpha_{n}\right)\left\|x_{n}-p\right\|^{2}+\alpha_{n}\left[2 \beta_{n}\left\|z_{n}-S z_{n}\right\|\left\|x_{n+1}-p\right\|\right. \\
& \left.+2\left\langle p, p-x_{n+1}\right\rangle+\frac{3 M \theta_{n}}{\alpha_{n}}\left\|x_{n}-x_{n-1}\right\|\right], \quad \forall n \geq n_{0} .
\end{aligned}
$$

Claim 4. $\left\{\left\|x_{n}-p\right\|^{2}\right\}$ converges to zero by considering two possible cases on $\left\{\left\|x_{n}-p\right\|^{2}\right\}$.

Case 1, There exists an $N \in \mathbb{N}$ such that $\left\|x_{n+1}-p\right\|^{2} \leq\left\|x_{n}-p\right\|^{2}$ for all $n \geq N$. This implies that $\lim _{n \rightarrow \infty}\left\|x_{n}-p\right\|^{2}$ exists. From Claim 2, Conditions (C5), (C9), and $\lim _{n \rightarrow \infty} \delta_{n}^{*}>0$, we obtain

$$
\lim _{n \rightarrow \infty}\left\|w_{n}-y_{n}\right\|=0, \lim _{n \rightarrow \infty}\left\|z_{n}-S z_{n}\right\|=0, \lim _{n \rightarrow \infty}\left\|z_{n}-y_{n}\right\|=0 .
$$

As stated in Claim 4 of Theorem 3.2, we can also obtain that

$$
\lim _{n \rightarrow \infty}\left\|z_{n}-w_{n}\right\|=0, \lim _{n \rightarrow \infty}\left\|x_{n}-w_{n}\right\|=0, \lim _{n \rightarrow \infty}\left\|z_{n}-x_{n}\right\|=0 .
$$


From (3.46) and the hypothesis on $\left\{\beta_{n}\right\}$, one has

$$
\lim _{n \rightarrow \infty} \beta_{n}\left\|z_{n}-S z_{n}\right\|\left\|x_{n+1}-p\right\|=0 .
$$

Combining (3.46), (3.47), and the hypothesis on $\left\{\alpha_{n}\right\}$, we have

$$
\left\|x_{n+1}-x_{n}\right\| \leq\left\|z_{n}-x_{n}\right\|+\alpha_{n}\left\|z_{n}\right\|+\beta_{n}\left\|z_{n}-S z_{n}\right\| \rightarrow 0 \text { as } n \rightarrow \infty .
$$

As declared in Case 1 of Theorem 3.2, we can obtain that $z \in \operatorname{EP}(f) \cap \mathrm{F}(S)$. According to the definition of $p$, one sees that $p=P_{\mathrm{EP}(f) \cap \mathrm{F}(S)}(0)$, which together with $z \in \operatorname{EP}(f) \cap \mathrm{F}(S)$ and (2.3) gives that

$$
\limsup _{n \rightarrow \infty}\left\langle p, p-x_{n}\right\rangle=\langle p, p-z\rangle \leq 0 .
$$

By using (3.49) and (3.50), we obtain

$$
\limsup _{n \rightarrow \infty}\left\langle p, p-x_{n+1}\right\rangle \leq 0 \text {. }
$$

Combining Claim 3, the hypothesis on $\left\{\alpha_{n}\right\}$, (3.48), (3.51), and Remark 3.2 (i), we deduce from Lemma 2.4 that $\lim _{n \rightarrow \infty}\left\|x_{n+1}-p\right\|^{2}=0$. That is, $x_{n} \rightarrow p$ as $n \rightarrow \infty$.

Case 2. There exists a subsequence $\left\{\left\|x_{n_{j}}-p\right\|^{2}\right\}$ of $\left\{\left\|x_{n}-p\right\|^{2}\right\}$ such that $\left\|x_{n_{j}}-p\right\|^{2}<$ $\left\|x_{n_{j}+1}-p\right\|^{2}$ for all $j \in \mathbb{N}$. By Claim 2 and (3.29), one has

$$
\begin{aligned}
& \delta_{m_{k}}^{*}\left(\left\|y_{m_{k}}-w_{m_{k}}\right\|^{2}+\left\|z_{m_{k}}-y_{m_{k}}\right\|^{2}\right)+\beta_{m_{k}}\left[(1-\lambda)-\beta_{m_{k}}\right]\left\|z_{m_{k}}-S z_{m_{k}}\right\|^{2} \\
\leq & \left\|x_{m_{k}}-p\right\|^{2}-\left\|x_{m_{k}+1}-p\right\|^{2}+\alpha_{m_{k}} M_{4} \leq \alpha_{m_{k}} M_{4} .
\end{aligned}
$$

From the hypothesis on $\left\{\beta_{n}\right\}$ and Remark 3.3, one obtains

$$
\lim _{k \rightarrow \infty}\left\|w_{m_{k}}-y_{m_{k}}\right\|=0, \lim _{k \rightarrow \infty}\left\|z_{m_{k}}-y_{m_{k}}\right\|=0, \lim _{k \rightarrow \infty}\left\|z_{m_{k}}-S z_{m_{k}}\right\|=0 .
$$

As proved in the first case, we can obtain that $\limsup _{k \rightarrow \infty}\left\langle p, p-x_{m_{k}+1}\right\rangle \leq 0$. From Claim 3 and (3.29), we have

$$
\begin{aligned}
\left\|x_{m_{k}+1}-p\right\|^{2} \leq & \left(1-\alpha_{m_{k}}\right)\left\|x_{m_{k}+1}-p\right\|^{2}+\alpha_{m_{k}}\left[2 \beta_{m_{k}}\left\|z_{m_{k}}-S z_{m_{k}}\right\|\left\|x_{m_{k}+1}-p\right\|\right. \\
& \left.+2\left\langle p, p-x_{m_{k}+1}\right\rangle+\frac{3 M \theta_{m_{k}}}{\alpha_{m_{k}}}\left\|x_{m_{k}}-x_{m_{k}-1}\right\|\right], \quad \forall m_{k} \geq n_{0},
\end{aligned}
$$

which together with (3.29) implies that

$$
\begin{aligned}
\left\|x_{k}-p\right\|^{2} & \leq\left\|x_{m_{k}+1}-p\right\|^{2} \\
& \leq 2 \beta_{m_{k}}\left\|z_{m_{k}}-S z_{m_{k}}\right\|\left\|x_{m_{k}+1}-p\right\|+2\left\langle p, p-x_{m_{k}+1}\right\rangle+\frac{3 M \theta_{m_{k}}}{\alpha_{m_{k}}}\left\|x_{m_{k}}-x_{m_{k}-1}\right\| .
\end{aligned}
$$

Thus, we conclude that $\lim \sup _{k \rightarrow \infty}\left\|x_{k}-p\right\|^{2} \leq 0$, that is $x_{k} \rightarrow p$ as $k \rightarrow \infty$. This completes the proof of Theorem 3.3.

Remark 3.4. We have the following observations for Algorithms 3.1-3.3.

(1) The algorithms presented in this paper use a new non-monotonic step size criterion (see (3.3)), which allows them to work without the prior knowledge of the Lipschitz constants for the bifunction $f$. Our results improve the algorithms in [7, 8, 9, 20] that use a fixed step size, the algorithms in $[23,24]$ that use the Armijo line search rule, and the algorithms in $[21,25]$ that use a non-increasing step size criterion. 
(2) It is worth noting that the suggested algorithms differ from the subgradient extragradient method proposed by Censor, Gibali and Reich $[16,17,18]$ in the calculation of $z_{n}$. Specifically, we replace the step size $\lambda_{n}$ in the algorithm proposed by Censor, Gibali and Reich $[16,17,18]$ with $\delta \lambda_{n}$ (where $\delta$ is a positive constant satisfying certain conditions) in our algorithms when calculating $z_{n}$. Notice that our schemes can obtain a faster convergence speed and a higher accuracy when $\delta$ is chosen to a suitable value (see the numerical experiments in Section 5).

(3) Our algorithms only require the computation of the strongly convex problem on the feasible set once in each iteration, which improves the computational speed of the algorithms proposed in $[7,8,9,20]$ that require the computation of the strongly convex problem on the feasible set twice. On the other hand, the inertial term, which is one of the techniques to speed up the convergence speed, is also embedded in the proposed algorithms. Numerical experimental results show that the appropriate inertial parameter plays an active role in the convergence speed and accuracy of our algorithms (see Section 5).

(4) It should be noted that the algorithms presented in $[8,23]$ are based on the hybrid (or shrinking) projection-type method to obtain strong convergence theorems in infinite-dimensional Hilbert spaces, while the proposed algorithms achieve strong convergence through the Mann-type method and the viscosity-type method. Our algorithms may be preferable to the projection-based methods $[8,23]$ in infinite-dimensional spaces due to the fact that the values of the projections are not easy to compute.

(5) We can obtain three new iterative schemes for solving the equilibrium problem when $S=I$, where $I$ is the identity operator. These results improve and generalize many algorithms used in the literature for solving equilibrium problems (see, e.g., $[11,12,13,14,15,19,22,26]$ ), based on the following five facts: (i) our algorithms improve the computational efficiency of extragradient-type algorithms $[11,14,26]$ due to the fact that only one optimization problem in the feasible set needs to be computed in each iteration; (ii) our algorithms include a pseudomonotone bifunction, which extends the results used in $[13,22,26]$ for solving monotone or strongly pseudomonotone equilibrium problems; (iii) our algorithms apply a new non-monotonic step size criterion, which is different from the non-summable and non-increasing step sizes used in [12, 15, 19, 22, 26]; (iv) our algorithms embed inertial terms to accelerate the convergence speed of the algorithms used; and (v) our algorithms obtain strong convergence theorems in infinite-dimensional Hilbert spaces, which is more preferable to the weakly convergent results proposed in $[12,14,15]$.

3.4. The Algorithm 3.4 and its linear convergence. In this subsection, we state the last iterative scheme proposed in this paper and analyze its convergence rate (see Algorithm 3.4 below). It is worth noting that the bifunction $f$ in Algorithm 3.4 is $\sigma$-strongly pseudomonotone on $C$. Now, we show the details of the proposed scheme in Algorithm 3.4.

The following results can be obtained with the help of the method presented in [19].

Theorem 3.4. Assume that the bifunction $f: \mathscr{H} \times \mathscr{H} \rightarrow \mathbb{R}$ is $\sigma$-strongly pseudomonotone on $C$ and satisfies Assumption (A2). Take a constant $\gamma \in(0,1)$. Suppose that the inertial parameter $\theta$ satisfies the following condition

$$
\theta \leq \frac{\eta \phi}{\eta \phi+2 \eta+\phi}
$$




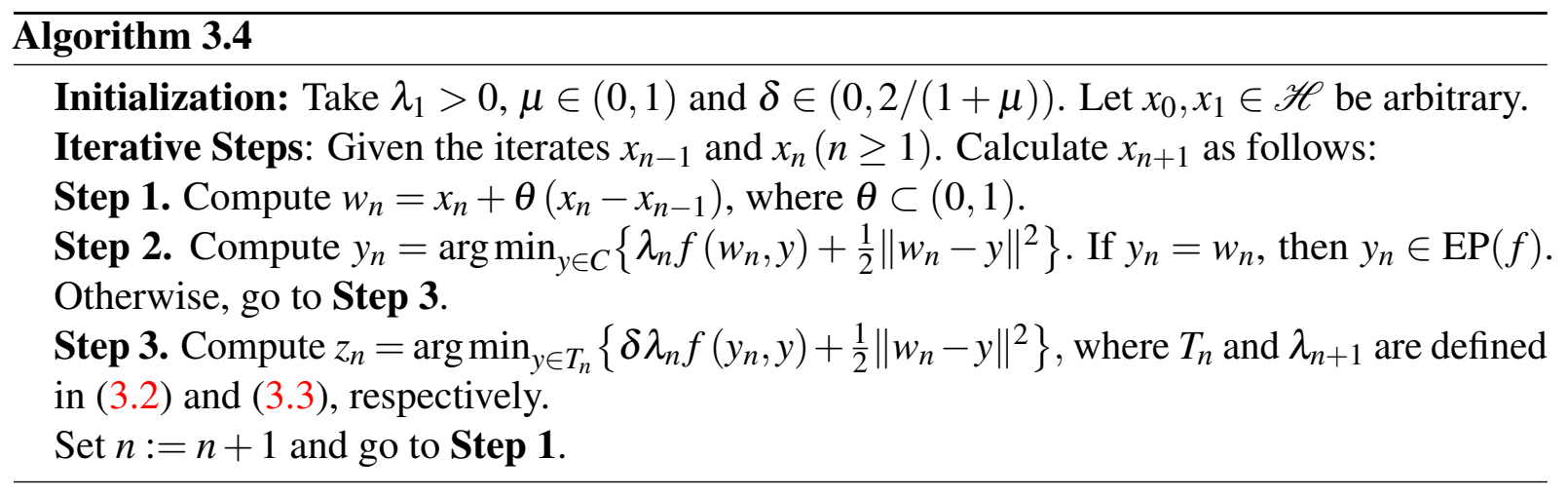

where $\eta:=1-\chi, \phi:=\chi(1-\gamma), \chi:=\min \left\{\frac{1}{2} \delta_{n}^{\dagger} \gamma, \delta \lambda \sigma\right\}, \lambda:=\lim _{n \rightarrow \infty} \lambda_{n}, \delta_{n}^{\dagger}:=\lim _{n \rightarrow \infty} \delta_{n}^{*}$ and $\delta_{n}^{*}$ is defined in Lemma 3.2. Then the sequence $\left\{x_{n}\right\}$ generated by Algorithm 3.4 converges to the unique solution $p$ of problem $\mathrm{EP}(f)$ with a R-linear rate.

Proof. We divided the proof into three steps.

Step 1. For all $p \in \operatorname{EP}(f)$, we show that

$$
\left\|x_{n+1}-p\right\|^{2} \leq \eta\left\|w_{n}-p\right\|^{2}-\phi\left\|x_{n+1}-w_{n}\right\|^{2}, \forall n \geq n_{0},
$$

where $\eta:=1-\chi \in(0,1)$ and $\phi:=\chi(1-\gamma) \in(0,1)$. Indeed, taking $y:=p$ in (3.6), we have

$$
\delta \lambda_{n}\left(f\left(y_{n}, p\right)-f\left(y_{n}, x_{n+1}\right)\right) \geq\left\langle w_{n}-x_{n+1}, p-x_{n+1}\right\rangle .
$$

From $p \in \operatorname{EP}(f)$ and $y_{n} \in C$, one obtains $f\left(p, y_{n}\right) \geq 0$, which together with the strong pseudomonotonicity of bifuntion $f$ yields that $f\left(y_{n}, p\right) \leq-\sigma\left\|y_{n}-p\right\|^{2}$. From (3.53), we see that

$$
\begin{aligned}
-\delta \lambda_{n} f\left(y_{n}, x_{n+1}\right) & \geq-\delta \lambda_{n} f\left(y_{n}, p\right)+\left\langle w_{n}-x_{n+1}, p-x_{n+1}\right\rangle \\
& \geq \delta \lambda_{n} \sigma\left\|y_{n}-p\right\|^{2}+\left\langle w_{n}-x_{n+1}, p-x_{n+1}\right\rangle .
\end{aligned}
$$

Combining (3.54) and the similar proof in (3.9)-(3.13), we obtain

$$
\left\|x_{n+1}-p\right\|^{2} \leq\left\|w_{n}-p\right\|^{2}-\delta_{n}^{*}\left(\left\|y_{n}-w_{n}\right\|^{2}+\left\|x_{n+1}-y_{n}\right\|^{2}\right)-2 \delta \lambda_{n} \sigma\left\|y_{n}-p\right\|^{2},
$$

where $\delta_{n}^{*}$ is defined in Lemma 3.2. It follows from Remark 3.3 that $\delta_{n}^{*}>0$ for all $n \geq n_{0}$. By means of (3.55) and the Cauchy-Schwartz Inequality $\left(2\|a\|^{2}+2\|b\|^{2} \geq\|a+b\|^{2}\right)$, we obtain for all $n \geq n_{0}$ that

$$
\begin{aligned}
\left\|x_{n+1}-p\right\|^{2} \leq & \left\|w_{n}-p\right\|^{2}-\delta_{n}^{*} \gamma\left\|y_{n}-w_{n}\right\|^{2}-2 \delta \lambda_{n} \sigma\left\|y_{n}-p\right\|^{2} \\
& -\delta_{n}^{*}(1-\gamma)\left[\left\|y_{n}-w_{n}\right\|^{2}+\left\|x_{n+1}-y_{n}\right\|^{2}\right] \\
\leq & \left\|w_{n}-p\right\|^{2}-\delta_{n}^{*} \gamma\left\|y_{n}-w_{n}\right\|^{2}-2 \delta \lambda_{n} \sigma\left\|y_{n}-p\right\|^{2} \\
& -\frac{1}{2} \delta_{n}^{*}(1-\gamma)\left\|x_{n+1}-w_{n}\right\|^{2}, \forall \gamma \in(0,1) .
\end{aligned}
$$

Take $\chi:=\min \left\{\frac{1}{2} \delta_{n}^{\dagger} \gamma, \delta \lambda \sigma\right\}$, where $\delta_{n}^{\dagger}:=2-\delta-\delta \mu$ when $\delta \in[1,2 /(1+\mu))$ and $\delta_{n}^{\dagger}:=\delta-\delta \mu$ when $\delta \in(0,1)$, and $\lambda:=\lim _{n \rightarrow \infty} \lambda_{n}$. Note that $\lim _{n \rightarrow \infty} \delta_{n}^{*}=\delta_{n}^{\dagger}$. Hence, there exists $N \geq n_{0}$ such that for all $n \geq N$,

$$
\frac{1}{2} \delta_{n}^{*}(1-\gamma) \geq \frac{1}{2} \delta_{n}^{\dagger}(1-\gamma) \gamma \geq \chi(1-\gamma), \delta_{n}^{*} \gamma \geq 2 \chi, \text { and } 2 \delta \lambda_{n} \sigma \geq 2 \chi
$$


From (3.56) and the Cauchy-Schwartz Inequality, we obtain

$$
\begin{aligned}
\left\|x_{n+1}-p\right\|^{2} & \leq\left\|w_{n}-p\right\|^{2}-2 \chi\left(\left\|y_{n}-w_{n}\right\|^{2}+\left\|y_{n}-p\right\|^{2}\right)-\chi(1-\gamma)\left\|x_{n+1}-w_{n}\right\|^{2} \\
& \leq \eta\left\|w_{n}-p\right\|^{2}-\phi\left\|x_{n+1}-w_{n}\right\|^{2}, \forall n \geq N .
\end{aligned}
$$

where $\eta:=1-\chi \in(0,1)$ and $\phi:=\chi(1-\gamma) \in(0,1)$.

Step 2. For all $n \geq N$, we show that

$$
\begin{aligned}
& \left\|x_{n+1}-p\right\|^{2}-\eta \theta\left\|x_{n}-p\right\|^{2}+\phi(1-\theta)\left\|x_{n+1}-x_{n}\right\|^{2} \\
\leq & \eta\left[\left\|x_{n}-p\right\|^{2}-\theta\left\|x_{n-1}-p\right\|^{2}+\phi(1-\theta)\left\|x_{n}-x_{n-1}\right\|^{2}\right] .
\end{aligned}
$$

From the definition of $w_{n}$ and (2.2), one has

$$
\begin{aligned}
\left\|w_{n}-p\right\|^{2} & =\left\|(1+\theta)\left(x_{n}-p\right)-\theta\left(x_{n-1}-p\right)\right\|^{2} \\
& =(1+\theta)\left\|x_{n}-p\right\|^{2}-\theta\left\|x_{n-1}-p\right\|^{2}+\theta(1+\theta)\left\|x_{n}-x_{n-1}\right\|^{2},
\end{aligned}
$$

and

$$
\begin{aligned}
\left\|x_{n+1}-w_{n}\right\|^{2} & =\left\|x_{n+1}-x_{n}-\theta\left(x_{n}-x_{n-1}\right)\right\|^{2} \\
& \geq\left\|x_{n+1}-x_{n}\right\|^{2}+\theta^{2}\left\|x_{n}-x_{n-1}\right\|^{2}-2 \theta\left\|x_{n+1}-x_{n}\right\|\left\|x_{n}-x_{n-1}\right\| \\
& \geq\left\|x_{n+1}-x_{n}\right\|^{2}+\theta^{2}\left\|x_{n}-x_{n-1}\right\|^{2}-\theta\left\|x_{n+1}-x_{n}\right\|^{2}-\theta\left\|x_{n}-x_{n-1}\right\|^{2} \\
& =(1-\theta)\left\|x_{n+1}-x_{n}\right\|^{2}-\theta(1-\theta)\left\|x_{n}-x_{n-1}\right\|^{2} .
\end{aligned}
$$

Combining these inequalities with (3.57), we have

$$
\begin{aligned}
\left\|x_{n+1}-p\right\|^{2} \leq & \eta(1+\theta)\left\|x_{n}-p\right\|^{2}-\eta \theta\left\|x_{n-1}-p\right\|^{2}+\eta \theta(1+\theta)\left\|x_{n}-x_{n-1}\right\|^{2} \\
& -\phi(1-\theta)\left\|x_{n+1}-x_{n}\right\|^{2}+\phi \theta(1-\theta)\left\|x_{n}-x_{n-1}\right\|^{2} .
\end{aligned}
$$

This is equivalent to

$$
\begin{aligned}
& \left\|x_{n+1}-p\right\|^{2}-\eta \theta\left\|x_{n}-p\right\|^{2}+\phi(1-\theta)\left\|x_{n+1}-x_{n}\right\|^{2} \\
\leq & \eta\left[\left\|x_{n}-p\right\|^{2}-\theta\left\|x_{n-1}-p\right\|^{2}+\phi(1-\theta)\left\|x_{n}-x_{n-1}\right\|^{2}\right] \\
& -(\eta \phi(1-\theta)-\eta \theta(1+\theta)-\phi \theta(1-\theta))\left\|x_{n}-x_{n-1}\right\|^{2} .
\end{aligned}
$$

Take $\Omega_{n}=\eta \phi(1-\theta)-\eta \theta(1+\theta)-\phi \theta(1-\theta)$. Note that $\Omega_{n} \geq 0$ for all $n \geq N$. Indeed, since $\theta \in[0,1)$, one obtains $1+\theta \leq 2$ and $\theta(1-\theta) \leq \theta$. It follows from (3.52) that

$$
\Omega_{n} \geq \eta \phi(1-\theta)-2 \eta \theta-\phi \theta=\eta \phi-\theta(\eta \phi+2 \eta+\phi) \geq 0 .
$$

Thus we can obtain that (3.58) holds.

Step 3. Finally, we need to prove that $\left\{x_{n}\right\}$ converges to the unique solution of problem $\operatorname{EP}(f)$ with a $R$-linear rate. For convenience, we take

$$
\Gamma_{n}:=\left\|x_{n}-p\right\|^{2}-\theta\left\|x_{n-1}-p\right\|^{2}+\phi(1-\theta)\left\|x_{n}-x_{n-1}\right\|^{2}, \forall n \geq N .
$$

Note that $\Gamma_{n} \geq 0, \forall n>N$. Indeed, it follows from the definition of $\Gamma_{n}$ that

$$
\begin{aligned}
\Gamma_{n} & =(1-\phi(1-\theta))\left\|x_{n}-p\right\|^{2}+\phi(1-\theta)\left(\left\|x_{n}-p\right\|^{2}+\left\|x_{n}-x_{n-1}\right\|^{2}\right)-\theta\left\|x_{n-1}-p\right\|^{2} \\
& \geq(1-\phi(1-\theta))\left\|x_{n}-p\right\|^{2}+\left(\frac{\phi(1-\theta)-2 \theta}{2}\right)\left\|x_{n-1}-p\right\|^{2} .
\end{aligned}
$$


From $\phi \in(0,1)$ and $\theta \in(0,1)$, one obtains $(1-\phi(1-\theta)) \in(0,1)$. By virtue of (3.52), one sees that

$$
\theta \leq \frac{\eta \phi}{\eta \phi+2 \eta+\phi} \leq \frac{\phi}{2+\phi}
$$

which means that

$$
\phi(1-\theta)-2 \theta \geq \phi-(\phi+2) \frac{\phi}{2+\phi} \geq 0 .
$$

Therefore, we deduce that $\Gamma_{n} \geq 0$ for all $n>N$. On the other hand, combining the definition of $\Gamma_{n}$ and $\eta \in(0,1)$, we have

$$
\begin{aligned}
\Gamma_{n+1} & =\left\|x_{n+1}-p\right\|^{2}-\theta\left\|x_{n}-p\right\|^{2}+\phi(1-\theta)\left\|x_{n+1}-x_{n}\right\|^{2} \\
& \leq\left\|x_{n+1}-p\right\|^{2}-\eta \theta\left\|x_{n}-p\right\|^{2}+\phi(1-\theta)\left\|x_{n+1}-x_{n}\right\|^{2},
\end{aligned}
$$

which together with (3.58) implies that $\Gamma_{n+1} \leq \eta \Gamma_{n}, \forall n \geq N$. Therefore, we conclude that

$$
(1-\phi(1-\theta))\left\|x_{n}-p\right\|^{2} \leq \Gamma_{n} \leq \eta^{n-N} \Gamma_{N} .
$$

That is,

$$
\left\|x_{n}-p\right\|^{2} \leq \frac{\Gamma_{N}}{(1-\phi(1-\theta)) \eta^{N}} \eta^{n}
$$

which implies that $\left\{x_{n}\right\}$ converges $R$-linearly to $p$. The proof is completed.

\section{ApPlicAtion to VARIATIONAL INEQUALITy PROBLEMS}

Let $C$ be a nonempty, closed, and convex subset of a real Hilbert space $\mathscr{H}$ with inner product $\langle\cdot, \cdot\rangle$ and induced norm $\|\cdot\|$. For all $x, y \in C$, a mapping $A: \mathscr{H} \rightarrow \mathscr{H}$ is said to be (i) L-Lipschitz continuous if there exists a positive constant $L$ such that $\|A x-A y\| \leq L\|x-y\|$; (ii) monotone if $\langle A x-A y, x-y\rangle \geq 0$; (iii) pseudomonotone if $\langle A x, y-x\rangle \geq 0$ implies that $\langle A y, y-x\rangle \geq 0$; (iv) strongly monotone if there exists a positive constant $L$ such that $\langle A x-A y, x-y\rangle \geq L\|x-y\|^{2}$; (v) sequentially weakly continuous if for each sequence $\left\{x_{n}\right\}$ converging weakly to $x$ implies that $\left\{A x_{n}\right\}$ converges weakly to $A x$. Recall that the variational inequality problem (shortly, VI) for operator $A$ on $C$ is stated as follows:

$$
\text { find } x^{*} \in C \text { such that }\left\langle A x^{*}, x-x^{*}\right\rangle \geq 0, \quad \forall x \in C \text {. }
$$

The solution set of the problem (VI) is denoted by $\mathrm{VI}(A)$.

Let $f(x, y)=\langle A x, y-x\rangle$ for all $x, y \in \mathscr{H}$, where $A: \mathscr{H} \rightarrow \mathscr{H}$ is an operator. If $A$ is $L$-Lipschitz continuous, then $f$ satisfies the Lipschitz-type condition and $L=2 c_{1}=2 c_{2}$. Indeed, by the definition of the Lipschitz-type condition of $f$, one sees that

$$
\begin{aligned}
c_{1}\|x-y\|^{2}+c_{2}\|y-z\|^{2} & \geq\langle A x, z-x\rangle-\langle A x, y-x\rangle-\langle A y, z-y\rangle \\
& =\langle A x-A y, z-y\rangle \leq\|A x-A y\|\|z-y\| .
\end{aligned}
$$

Since operator $A$ is $L$-Lipschitz continuous, one sees that

$$
\|A x-A y\|\|z-y\| \leq L\|x-y\|\|z-y\| \leq(L / 2)\left(\|x-y\|^{2}+\|y-z\|^{2}\right) .
$$

Therefore, we can deduce that $L=2 c_{1}=2 c_{2}$. Furthermore, it is easy to check that bifunction $f$ is pseudomonotone when operator $A$ is pseudomonotone. 
Next, we derive the formulas for $y_{n}$ and $z_{n}$ in the variational inequality problem. By the definition of $y_{n}$ defined in Algorithm 3.1, one has

$$
\begin{aligned}
y_{n} & =\arg \min _{y \in C}\left\{\lambda_{n} f\left(w_{n}, y\right)+\frac{1}{2}\left\|w_{n}-y\right\|^{2}\right\} \\
& =\arg \min _{y \in C}\left\{\lambda_{n}\left\langle A w_{n}, y-w_{n}\right\rangle+\frac{1}{2}\left\|w_{n}-y\right\|^{2}+\frac{\lambda_{n}^{2}}{2}\left\|A w_{n}\right\|^{2}\right\} \\
& =\arg \min _{y \in C}\left\{\frac{1}{2}\left\|w_{n}-y-\lambda_{n} A w_{n}\right\|^{2}\right\}=P_{C}\left(w_{n}-\lambda_{n} A w_{n}\right) .
\end{aligned}
$$

Using a similar approach, we can obtain $z_{n}=P_{T_{n}}\left(w_{n}-\delta \lambda_{n} A y_{n}\right)$ from the definition of $z_{n}$ in Algorithm 3.1. From (3.9) and $f(x, y)=\langle A x, y-x\rangle$, one obtains

$$
\left\langle A w_{n}, y-w_{n}\right\rangle-\left\langle A w_{n}, y_{n}-w_{n}\right\rangle \geq\left\langle v_{n}, y-y_{n}\right\rangle, \quad \forall y \in \mathscr{H},
$$

which implies that $\left\langle A w_{n}-v_{n}, y-y_{n}\right\rangle \geq 0$ for all $y \in \mathscr{H}$. It follows form the definition of $T_{n}$ in Algorithm 3.1 that

$$
\begin{aligned}
0 & \geq\left\langle w_{n}-\lambda_{n} A w_{n}-y_{n}, y-y_{n}\right\rangle+\lambda_{n}\left\langle A w_{n}-v_{n}, y-y_{n}\right\rangle \\
& \geq\left\langle w_{n}-\lambda_{n} A w_{n}-y_{n}, y-y_{n}\right\rangle .
\end{aligned}
$$

As a result, we can choose $T_{n}$ as $T_{n}=\left\{y \in \mathscr{H} \mid\left\langle w_{n}-\lambda_{n} A w_{n}-y_{n}, y-y_{n}\right\rangle \leq 0\right\}$. According to $f(x, y)=\langle A x, y-x\rangle$ and (3.3), we have

$$
\lambda_{n+1}=\left\{\begin{array}{l}
\min \left\{\frac{\mu\left(\left\|w_{n}-y_{n}\right\|^{2}+\left\|z_{n}-y_{n}\right\|^{2}\right)}{2\left\langle A w_{n}-A y_{n}, z_{n}-y_{n}\right\rangle}, \xi_{n} \lambda_{n}\right\}, \\
\text { if }\left\langle A w_{n}-A y_{n}, z_{n}-y_{n}\right\rangle>0 ; \\
\xi_{n} \lambda_{n}, \text { otherwise. }
\end{array}\right.
$$

Now, we can obtain the following corollaries by applying the results presented in Section 3.

Corollary 4.1. Assume that the solution set $\operatorname{VI}(A) \cap \mathrm{F}(S) \neq \emptyset$, and the operator $A: \mathscr{H} \rightarrow \mathscr{H}$ is pseudomonotone, L-Lipschitz continuous on $\mathscr{H}$, and sequentially weakly continuous on $C$. Take $\theta>0, \lambda_{1}>0, \mu \in(0,1)$, and $\delta \in(0,2 /(1+\mu))$. Let $\theta_{n}$ and $\lambda_{n}$ be defined in (3.1) and (4.1), respectively. Let $x_{0}, x_{1} \in \mathscr{H}$ be arbitrary.

(1) Suppose that Conditions (C3)-(C6) hold. Let the sequence $\left\{x_{n}\right\}$ be generated by

$$
\left\{\begin{array}{l}
w_{n}=x_{n}+\theta_{n}\left(x_{n}-x_{n-1}\right), \\
y_{n}=P_{C}\left(w_{n}-\lambda_{n} A w_{n}\right), \\
z_{n}=P_{T_{n}}\left(w_{n}-\delta \lambda_{n} A y_{n}\right), \\
T_{n}=\left\{x \in \mathscr{H} \mid\left\langle w_{n}-\lambda_{n} A w_{n}-y_{n}, x-y_{n}\right\rangle \leq 0\right\} \\
t_{n}=\alpha_{n} \varphi\left(x_{n}\right)+\left(1-\alpha_{n}\right) z_{n}, \\
x_{n+1}=\chi_{n} z_{n}+\left(1-\chi_{n}\right) S t_{n} .
\end{array}\right.
$$

(2) Suppose that Conditions (C4), (C5), (C7), and (C8) hold. Let the sequence $\left\{x_{n}\right\}$ be generated by

$$
\left\{\begin{array}{l}
w_{n}, y_{n} \text { and } z_{n} \text { are the same as in (4.2), } \\
t_{n}=\left(1-\beta_{n}\right) z_{n}+\beta_{n} S z_{n} \\
x_{n+1}=\alpha_{n} \varphi\left(x_{n}\right)+\left(1-\alpha_{n}\right) t_{n}
\end{array}\right.
$$


(3) Suppose that Conditions (C5), (C7), and (C9) hold. Let the sequence $\left\{x_{n}\right\}$ be generated by

$$
\left\{\begin{array}{l}
w_{n}, y_{n} \text { and } z_{n} \text { are the same as in (4.2), } \\
x_{n+1}=\left(1-\alpha_{n}-\beta_{n}\right) z_{n}+\beta_{n} S z_{n}
\end{array}\right.
$$

Then the sequence $\left\{x_{n}\right\}$ generated by Algorithm (4.2) [or Algorithm (4.3), or Algorithm (4.4)] converges to $p \in \mathrm{VI}(A) \cap \mathrm{F}(S)$ in norm.

Corollary 4.2. Assume that the solution set $\mathrm{VI}(A) \neq \emptyset$, and the operator $A: \mathscr{H} \rightarrow \mathscr{H}$ is $\sigma$-strongly pseudomonotone on $C$, L-Lipschitz continuous on $\mathscr{H}$ and sequentially weakly continuous on $C$. Take $\lambda_{1}>0, \mu \in(0,1), \delta \in(0,2 /(1+\mu))$ and $\gamma \in(0,1)$. Let $\lambda_{n}$ be defined in (4.1). Suppose that the inertial parameter $\theta$ satisfies (3.52). Let the sequence $\left\{x_{n}\right\}$ be generated by

$$
\left\{\begin{array}{l}
w_{n}=x_{n}+\theta\left(x_{n}-x_{n-1}\right), \\
y_{n}=P_{C}\left(w_{n}-\lambda_{n} A w_{n}\right), \\
x_{n+1}=P_{T_{n}}\left(w_{n}-\delta \lambda_{n} A y_{n}\right), \\
T_{n}=\left\{x \in \mathscr{H} \mid\left\langle w_{n}-\lambda_{n} A w_{n}-y_{n}, x-y_{n}\right\rangle \leq 0\right\} .
\end{array}\right.
$$

Then the sequence $\left\{x_{n}\right\}$ generated by Algorithm (4.5) converges to the unique solution $p$ of problem $\mathrm{VI}(A)$ with a $R$-linear rate.

Remark 4.1. If $S=I$ in Algorithms (4.2)-(4.4), where $I$ is an identity operator, then we can obtain three new strongly convergent iterative schemes to solve the variational inequality problem (VI). It should be noted that we do not need to impose the sequentially weak continuity of the operator $A$ when it is monotone.

\section{NUMERICAL EXAMPLES}

In this section, we provide some numerical examples occurring in finite- and infinite-dimensional spaces and applications in optimal control problems to demonstrate the computational efficiency of the proposed algorithms compared to some previously known schemes. All the programs were implemented in MATLAB 2018a on a Intel(R) Core(TM) i5-8250S CPU @ 1.60GHz computer with RAM 8.00 GB.

\subsection{Theoretical examples.}

Example 5.1. Consider the linear operator $A: \mathbb{R}^{m} \rightarrow \mathbb{R}^{m}(m=20)$ in the form $A x=M x+q$, where $q \in \mathbb{R}^{m}$ and $M=N N^{\top}+Q+D, N$ is a $m \times m$ matrix, $Q$ is a $m \times m$ skew-symmetric matrix, and $D$ is a $m \times m$ diagonal matrix with its diagonal entries being nonnegative (hence $M$ is positive symmetric definite). The feasible set $C$ is given by $C=\left\{x \in \mathbb{R}^{m}:-2 \leq x_{i} \leq 5, i=1, \ldots, m\right\}$. It is clear that $A$ is monotone and Lipschitz continuous with constant $L=\|M\|$. Set $f(x, y)=$ $\langle A x, y-x\rangle$ and $S x=x$. We apply the proposed algorithms to solve the equilibrium problem (EP) with $f$ and $C$ given above. In this experiment, all entries of $N, Q$ are generated randomly in $[-2,2], D$ is generated randomly in $[0,2]$, and $q=\mathbf{0}$. It is easy to check that the solution to our problem is $x^{*}=\{\boldsymbol{0}\}$. The parameters of the proposed algorithms are set as follows. Take $\alpha_{n}=1 /(n+1), \varepsilon_{n}=100 /(n+1)^{2}, \lambda_{1}=1$ and $\mu=0.2$ for all the algorithms. Choose $\beta_{n}=0.1$ and $\varphi(x)=0.1 x$ for the proposed Algorithms 3.1 and 3.2. Select $\beta_{n}=0.5\left(1-\alpha_{n}\right)$ for the suggested Algorithm 3.3. The maximum number of iterations 500 is used as a common stopping criterion for all algorithms. We use $D_{n}=\left\|x_{n}-x^{*}\right\|$ to measure the error of the $n$-th iteration step. 
Next, we test the effect of different parameters $\theta, \delta$ and $\xi_{n}$ on the convergence behavior of the proposed algorithms. Figs. 1, 2 and 3 show the numerical behavior of the proposed algorithms for different parameters $\theta, \delta$ and $\xi_{n}$, respectively.

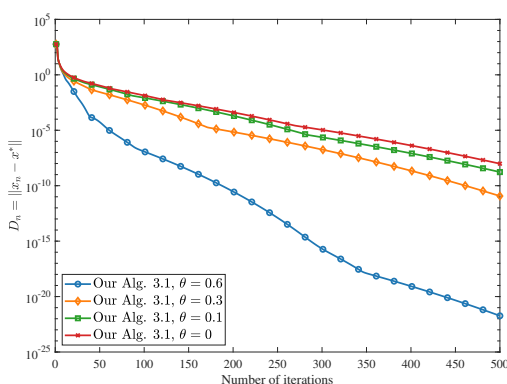

(a) Our Algorithm 3.1

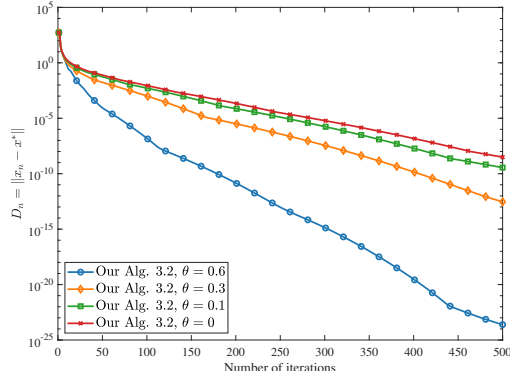

(b) Our Algorithm 3.2

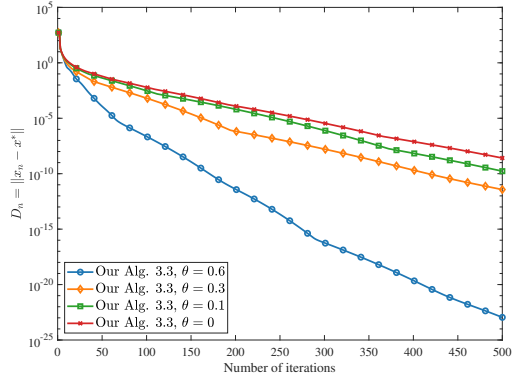

(c) Our Algorithm 3.3

FIGURE 1. Numerical behavior of the proposed algorithms with different $\theta$ for Example 5.1 (Set $\delta=1.5$ and $\xi_{n}=1+1 /(n+1)^{1.1}$ for the proposed algorithms)

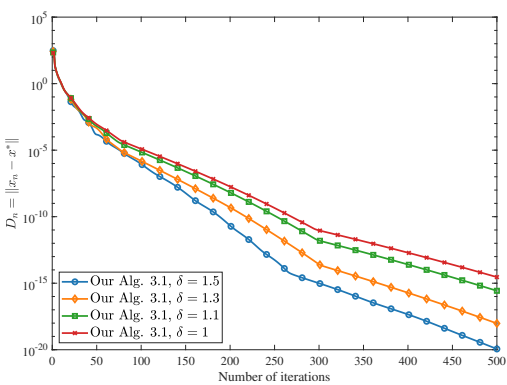

(a) Our Algorithm 3.1

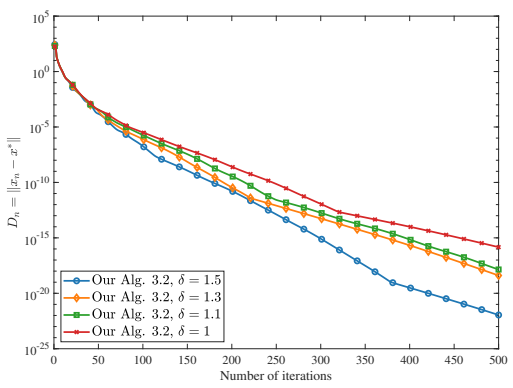

(b) Our Algorithm 3.2

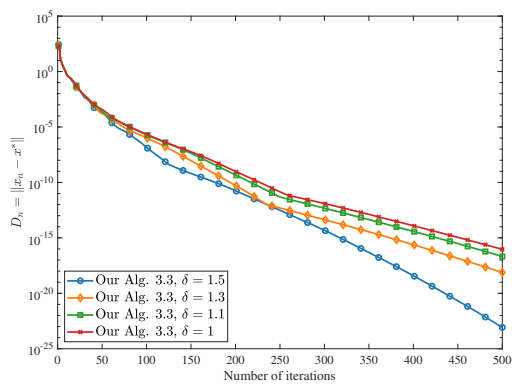

(c) Our Algorithm 3.3

FIGURE 2. Numerical behavior of the proposed algorithms with different $\delta$ for Example 5.1 (Set $\theta=0.6$ and $\xi_{n}=1+1 /(n+1)^{1.1}$ for the proposed algorithms)

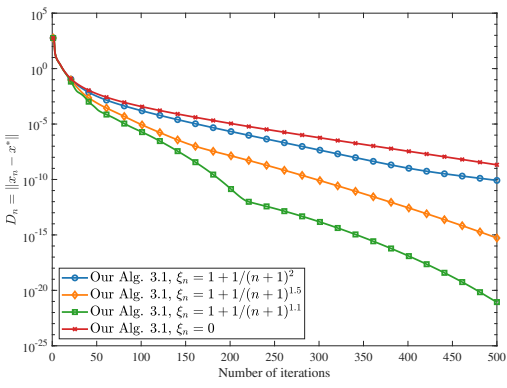

(a) Our Algorithm 3.1

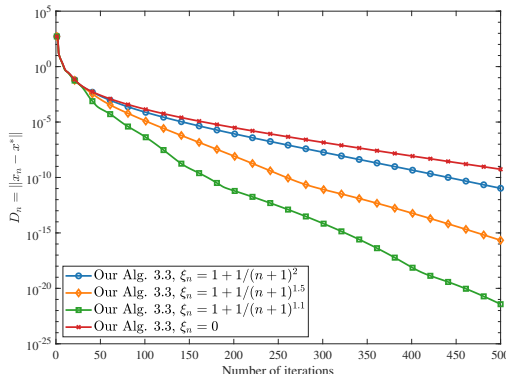

(b) Our Algorithm 3.2

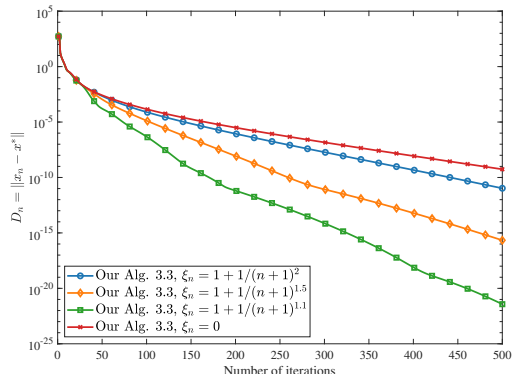

(c) Our Algorithm 3.3

FIGURE 3. Numerical behavior of the proposed algorithms with different $\xi_{n}$ for Example 5.1 (Set $\theta=0.6$ and $\delta=1.5$ for the proposed algorithms) 
Example 5.2. In this example, we consider the following bifunction $f: C \times C \rightarrow \mathbb{R}$ which comes from the Nash-Cournot equilibrium model in [11]:

$$
f(x, y)=\langle P x+Q y+q, y-x\rangle, \quad \forall x, y \in C,
$$

where $C \subset \mathbb{R}^{m}$ is the feasible set, $q \in \mathbb{R}^{m}$ and $P, Q \in \mathbb{R}^{m \times m}$ are two matrices of order $m$ such that $Q$ is symmetric, positive semidefinite and $Q-P$ is symmetric negative semidefinite (hence $f$ is monotone). In this case, the bifunction $f$ satisfies Condition (A2) with the Lipschitz-type constants $c_{1}=c_{2}=\frac{1}{2}\|Q-P\|$ (see [11, Lemma 6.2]). It can be checked that all the conditions (A1)-(A4) are satisfied. Let $C$ be given by $C=\left\{x \in \mathbb{R}^{m}:-5 \leq x_{i} \leq 5, i=1,2, \cdots, m\right\}$. In the next numerical experiments, we randomly generate the matrices $P$ and $Q$ by the following way. Two random orthogonal matrices $O_{1}=\operatorname{RandOrthMat}(m)$ and $O_{2}=\operatorname{RandOrthMat}(m)$ are generated using the RandOrthMat function ${ }^{1}$. Two diagonal matrices $A_{1}$ and $A_{2}$ with entries from $[0,2]$ and $[-2,0]$, respectively, are randomly generated. Then, a positive semi-infinite matrix $B_{1}=O_{1} A_{1} O_{1}^{\top}$ and a negative semi-infinite matrix $B_{2}=O_{2} A_{2} O_{2}^{\top}$ can be implemented. Finally, let $Q=B_{1}+B_{1}^{\top}, S=B_{2}+B_{2}^{\top}$ and $P=Q-S$. Vector $q$ is generated randomly and its elements are located at $(0,1)$.

We compare the proposed Algorithms 3.1-3.3 with some known iterative schemes in the literature [15, 19, 25], which including the Algorithm 3.1 proposed by Yang and Liu [25] (shortly, YL Alg. 3.1), the Algorithm 2.1 suggested by Shehu et al. [15] (shortly, SSTT Alg. 2.1), and the Algorithm 3.2 introduced by Thong et al. [19] (shortly, TCRC Alg. 3.2). The parameters of all algorithms are set as follows.

- Choose $\theta=0.2, \varepsilon_{n}=100 /(n+1)^{2}, \alpha_{n}=1 /(n+1), \mu=0.5, \lambda_{1}=0.1, \xi_{n}=1+1 /(n+1)^{1.1}$, $\delta=1.5$ and $S x=x$ for the proposed Algorithms 3.1-3.3. Take $\beta_{n}=0.5$ and $\varphi(x)=0.1 x$ for the proposed Algorithms 3.1 and 3.2. Set $\beta_{n}=0.5\left(1-\alpha_{n}\right)$ for the proposed Algorithm 3.3.

- Take $\alpha_{n}=1 /(n+1), \mu=0.5, \lambda_{1}=0.1, \beta_{n}=0.5$ and $\xi_{n}=1 /(n+1)^{1.1}$ for YL Alg. 3.1.

- Select $\alpha=0.1, \alpha_{n}=1 /(n+1), \mu=0.5, \lambda_{1}=0.1, \tau=0.3$ for SSTT Alg. 2.1.

- Pick $\theta=0.2, \varepsilon_{n}=100 /(n+1)^{2}, \alpha_{n}=1 /(n+1), \beta_{n}=0.5\left(1-\alpha_{n}\right), \mu=0.5$ and $\lambda_{1}=0.1$ for TCRC Alg. 3.2.

The initial values $x_{0}$ and $x_{1}$ are randomly generated by MATLAB function $\operatorname{rand}(m, 1)$ (rand $(m, 1)$ produces $m$ random numbers that lie within $(0,1)$ uniformly distributed). We use the maximum number of iterations 50 as a common stopping criterion for all algorithms. The function $D_{n}=\left\|x_{n}-x_{n-1}\right\|^{2}$ is used to measure the error of the $n$-th iteration due to the fact that we do not know the exact solution of the problem. We first test the numerical behavior of the proposed algorithm with different parameters $\delta$ and $\xi_{n}$, as shown in Fig. 4 and Table 1. Finally, the numerical results of all algorithms in different dimensions are shown in Fig. 5 and Table 2.

Example 5.3. Let $\mathscr{H}=L^{2}([0,1])$ be an infinite-dimensional Hilbert space with inner product $\langle x, y\rangle=\int_{0}^{1} x(t) y(t) \mathrm{d} t, \forall x, y \in \mathscr{H}$ and induced norm $\|x\|=\left(\int_{0}^{1}|x(t)|^{2} \mathrm{~d} t\right)^{1 / 2}, \forall x \in \mathscr{H}$. Let $r$ and $R$ be two positive real numbers such that $R /(k+1)<r / k<r<R$ for some $k>1$. Take the feasible set as $C=\{x \in \mathscr{H}:\|x\| \leq r\}$. The operator $A: \mathscr{H} \rightarrow \mathscr{H}$ is given by

$$
A x=(R-\|x\|) x, \quad \forall x \in \mathscr{H} .
$$

\footnotetext{
${ }^{1}$ The function "RandOrthMat" can be found at https://www.mathworks.com/matlabcentral/ fileexchange/11783-randorthmat
} 


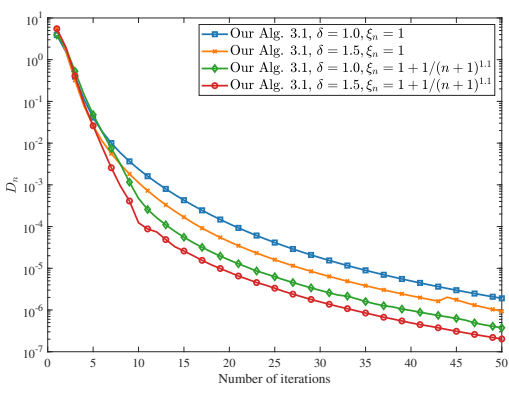

(a) Our Algorithm 3.1

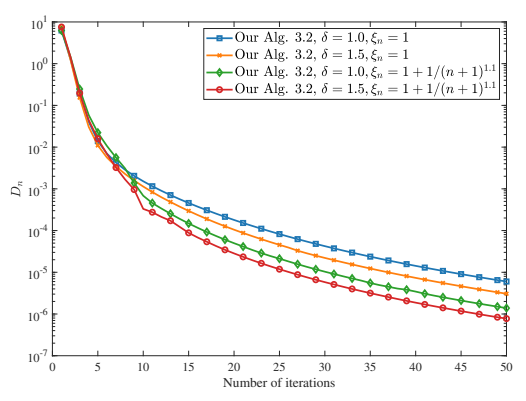

(b) Our Algorithm 3.2

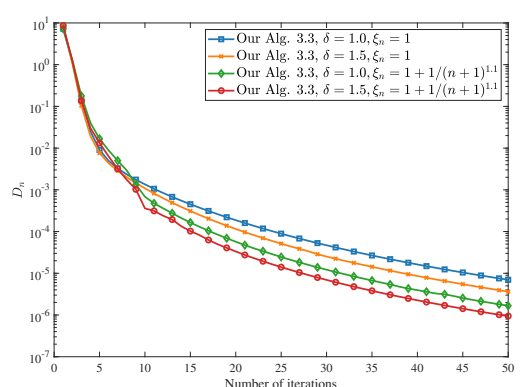

(c) Our Algorithm 3.3

FIGURE 4. Numerical behavior of the proposed algorithms with different parameters for Example 5.2

TABLE 1. Numerical results of the proposed algorithms with different parameters for Example 5.2

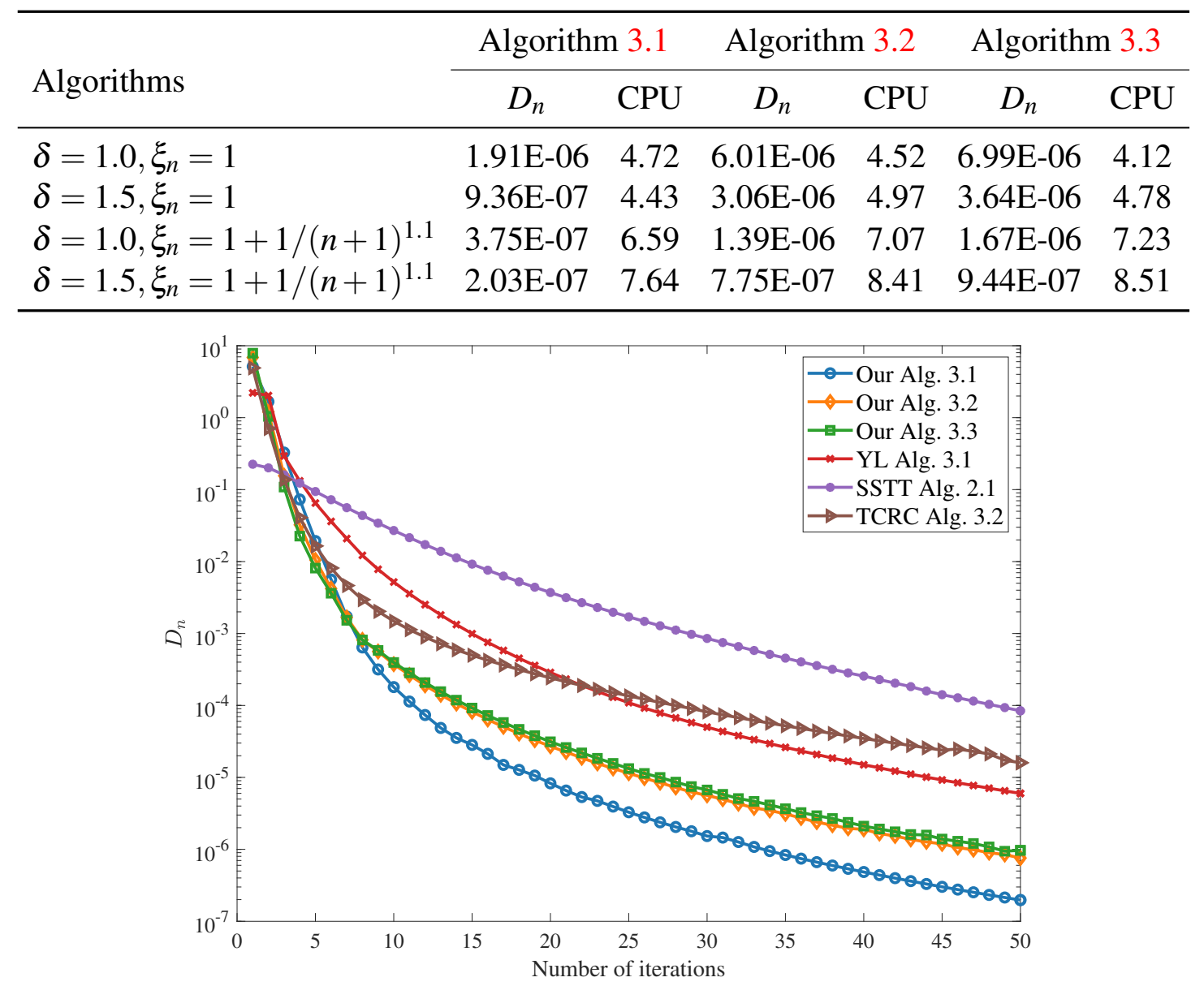

FIGURE 5. Numberical behavior of all algorithms for Example 5.2, $m=50$ 
TABLE 2. Numerical results of all algorithms in different dimensions for Example 5.2

\begin{tabular}{lcccccccc}
\hline \multirow{2}{*}{ Algorithms } & \multicolumn{2}{c}{$m=5$} & \multicolumn{2}{c}{$m=20$} & \multicolumn{2}{c}{$m=50$} & \multicolumn{2}{c}{$m=100$} \\
\cline { 2 - 9 } & $D_{n}$ & CPU & $D_{n}$ & CPU & $D_{n}$ & CPU & $D_{n}$ & CPU \\
\hline Our Alg. 3.1 & $1.38 \mathrm{E}-08$ & 2.70 & $4.51 \mathrm{E}-08$ & 3.18 & $1.97 \mathrm{E}-07$ & 6.91 & $2.36 \mathrm{E}-07$ & 12.59 \\
Our Alg. 3.2 & $5.03 \mathrm{E}-08$ & 2.49 & $1.74 \mathrm{E}-07$ & 3.32 & $7.58 \mathrm{E}-07$ & 6.65 & $3.98 \mathrm{E}-06$ & 12.05 \\
Our Alg. 3.3 & $6.05 \mathrm{E}-08$ & 2.50 & $2.11 \mathrm{E}-07$ & 3.41 & $9.70 \mathrm{E}-07$ & 6.66 & $1.51 \mathrm{E}-06$ & 12.63 \\
YL Alg. 3.1 & $4.70 \mathrm{E}-07$ & 2.74 & $1.99 \mathrm{E}-06$ & 3.94 & $5.99 \mathrm{E}-06$ & 6.90 & $1.08 \mathrm{E}-05$ & 13.20 \\
SSTT Alg. 2.1 & $2.79 \mathrm{E}-06$ & 1.99 & $3.50 \mathrm{E}-05$ & 2.39 & $8.43 \mathrm{E}-05$ & 3.78 & $1.84 \mathrm{E}-04$ & 6.96 \\
TCRC Alg. 3.2 & $7.74 \mathrm{E}-07$ & 2.62 & $3.59 \mathrm{E}-06$ & 2.20 & $1.60 \mathrm{E}-05$ & 3.43 & $2.32 \mathrm{E}-05$ & 6.93 \\
\hline
\end{tabular}

Note that the operator $A$ is pseudomonotone rather than monotone (see [40, Example 2]). We use the proposed Algorithms (4.2)-(4.4) to solve the variational inequality problem (VI) with $A$ and $C$ given above, and compare them with two strongly convergent inertial extragradient algorithms, which including the Algorithm 3.2 introduced by Thong, Hieu and Rassias [41] (shortly, THR Alg. 3.2) and the Algorithm (3.39) proposed by Tan, Liu and Qin [40] (shortly, TLQ Alg. (3.39)). For the experiment, we choose $R=1.5, r=1, k=1.1$. The solution of this problem is $x^{*}(t)=0$. The parameters of all algorithms are set as follows. Set $\alpha_{n}=1 /(n+1)$, $\theta=0.3, \varepsilon_{n}=100 /(n+1)^{2}, \varphi(x)=0.1 x, \mu=0.4$ and $\lambda_{1}=1$ for all algorithms. Take $\beta_{n}=0.5$ for the suggested Algorithm (4.2) and Algorithm (4.3). Select $\beta_{n}=0.5\left(1-\alpha_{n}\right)$ for the suggested Algorithm (4.4). Choose $\delta=1.5$ and $\xi_{n}=1+1 /(n+1)^{1.1}$ for the suggested Algorithm (4.2)Algorithm (4.4). The maximum number of iterations 50 is used as a common stopping criterion and $D_{n}=\left\|x_{n}(t)-x^{*}(t)\right\|$ is used to measure the error of the $n$-th iteration step of all algorithms. The numerical results of all algorithms with four initial values $x_{0}(t)=x_{1}(t)$ are shown in Fig. 6 and Table 3.

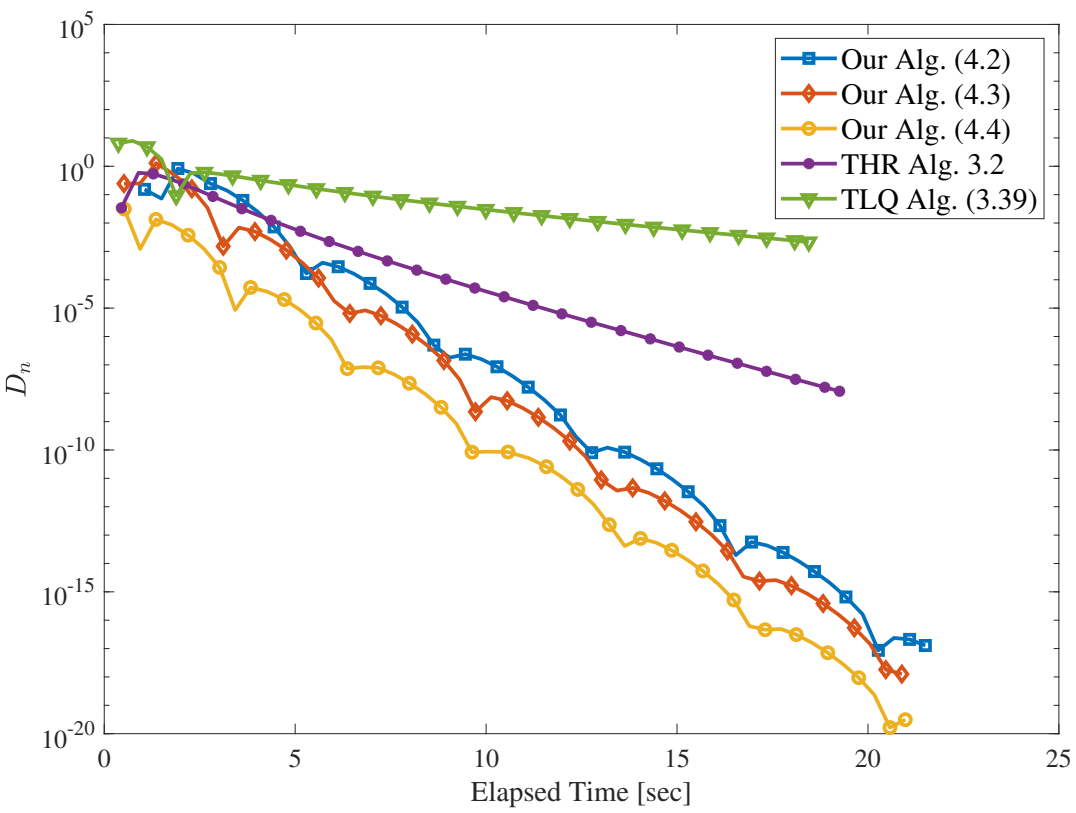

FIGURE 6. Numerical behavior of all algorithms for Example 5.3, $x_{0}(t)=x_{1}(t)=5 \cos (t)$ 
TABLE 3. Numerical results of all algorithms with different initial values for Example 5.3

\begin{tabular}{lcccccccc}
\hline \multirow{2}{*}{ Algorithms } & \multicolumn{2}{c}{$x_{1}(t)=5 t^{4}$} & \multicolumn{2}{c}{$x_{1}(t)=5 \mathrm{e}^{t}$} & \multicolumn{2}{c}{$x_{1}(t)=5 \log (t)$} & \multicolumn{2}{c}{$x_{1}(t)=5 \cos (t)$} \\
\cline { 2 - 9 } & $D_{n}$ & CPU & $D_{n}$ & CPU & $D_{n}$ & CPU & $D_{n}$ & CPU \\
\hline Our Alg. (4.2) & $3.26 \mathrm{E}-19$ & 21.38 & $8.95 \mathrm{E}-18$ & 21.55 & $2.25 \mathrm{E}-19$ & 21.79 & $1.28 \mathrm{E}-17$ & 21.50 \\
Our Alg. (4.3) & $1.44 \mathrm{E}-19$ & 20.55 & $2.83 \mathrm{E}-19$ & 20.98 & $3.16 \mathrm{E}-18$ & 21.02 & $1.26 \mathrm{E}-18$ & 20.89 \\
Our Alg. (4.4) & $1.03 \mathrm{E}-18$ & 20.79 & $1.50 \mathrm{E}-18$ & 21.00 & $9.48 \mathrm{E}-19$ & 21.02 & $3.11 \mathrm{E}-20$ & 20.98 \\
THR Alg. 3.2 & $2.64 \mathrm{E}-15$ & 18.97 & $3.20 \mathrm{E}-05$ & 19.23 & $6.68 \mathrm{E}-06$ & 19.33 & $1.17 \mathrm{E}-08$ & 19.26 \\
TLQ Alg. (3.39) & $5.29 \mathrm{E}-14$ & 18.24 & $6.95 \mathrm{E}-03$ & 18.43 & $1.46 \mathrm{E}-02$ & 18.83 & $2.15 \mathrm{E}-03$ & 18.45 \\
\hline
\end{tabular}

Remark 5.1. We have the following observations for Example 5.1-Example 5.3.

(1) The algorithms proposed in this paper can obtain a faster convergence speed when the appropriate values of the parameters $\theta_{n}, \delta$ and $\xi_{n}$ are chosen (see Fig. 1, Fig. 2, Fig. 3, Fig. 4, and Table 1).

(2) Our algorithms converge faster than some known ones in the literature [15, 19, 25, 40, 41] when performing the same stopping conditions, and these results are independent of the size of the dimension and the choice of the initial values (see Fig. 5, Fig. 6, Table 2, and Table 3). This shows that our algorithms are efficient and robust.

(3) Notice that the operator $A$ involved in Example 5.3 is pseudomonotone rather than monotone. The algorithms proposed in the literature (e.g., $[16,30]$ ) for solving monotone variational inequalities will not be available in this case. Therefore, the algorithms proposed in this paper are more useful and have a wider range of applications.

5.2. Application to optimal control problems. Next, we use the proposed algorithms to solve the variational inequality problem (VI) that appears in optimal control problems. Assume that $L_{2}\left([0, T], \mathbb{R}^{m}\right)$ represents the square-integrable Hilbert space with inner product $\langle p, q\rangle=$ $\int_{0}^{T}\langle p(t), q(t)\rangle \mathrm{d} t$ and norm $\|p\|=\sqrt{\langle p, p\rangle}$. The optimal control problem is described as follows:

$$
\left\{\begin{array}{l}
p^{*}(t) \in \operatorname{Argmin}\{g(p) \mid p \in V\}, \\
g(p)=\Phi(x(T)), \\
V=\left\{p(t) \in L_{2}\left([0, T], \mathbb{R}^{m}\right): p_{i}(t) \in\left[p_{i}^{-}, p_{i}^{+}\right], i=1,2, \ldots, m\right\}, \\
\text { s.t. } \dot{x}(t)=Q(t) x(t)+W(t) p(t), 0 \leq t \leq T, x(0)=x_{0},
\end{array}\right.
$$

where $g(p)$ means the terminal objective function, $\Phi$ is convex and differentiable defined on the attainability set, $p(t)$ denotes the control function, $V$ represents a set of feasible controls composed of $m$ piecewise continuous functions, $x(t)$ stands for the trajectory, and $Q(t) \in \mathbb{R}^{n \times n}$ and $W(t) \in \mathbb{R}^{n \times m}$ are given continuous matrices for every $t \in[0, T]$. By the solution of problem (5.1), we mean a control $p^{*}(t)$ and a corresponding (optimal) trajectory $x^{*}(t)$ such that its terminal value $x^{*}(T)$ minimizes objective function $g(p)$. It is known that the optimal control problem (5.1) can be transformed into a variational inequality problem (see [42,43]). We first use the classical Euler discretization method to decompose the optimal control problem (5.1) and then apply the proposed algorithms to solve the variational inequality problem corresponding to the discretized version of the problem (see $[42,43]$ for more details). 
We compare the suggested Algorithms (4.2)-(4.4) with THR Alg. 3.2 [41] and TLQ Alg. (3.39) [40]. The parameters of all the algorithms are set as follows.

- In the proposed Algorithms (4.2)-(4.4), we set $N=100, \theta=0.01, \varepsilon_{n}=10^{-4} /(n+1)^{2}$, $\alpha_{n}=10^{-4} /(n+1), \delta=1.5, \lambda_{1}=0.4, \mu=0.1, \xi_{n}=1+5 /(n+1)^{1.1}$ and $S x=x$. Take $\beta_{n}=0.5$ and $\varphi(x)=0.1 x$ for the suggested Algorithm (4.2) and Algorithm (4.3). Select $\beta_{n}=0.5\left(1-\alpha_{n}\right)$ for the suggested Algorithm (4.4).

- In the THR Alg. 3.2 and the TLQ Alg. (3.39), we choose $N=100, \theta=0.01, \varepsilon_{n}=$ $10^{-4} /(n+1)^{2}, \alpha_{n}=10^{-4} /(n+1), \lambda_{1}=0.4, \mu=0.1$ and $\varphi(x)=0.1 x$.

The initial controls $p_{0}(t)=p_{1}(t)$ are randomly generated in $[-1,1]$. The stopping criterion is either $D_{n}=\left\|p_{n+1}-p_{n}\right\| \leq 10^{-4}$ or reaching the maximum number of iterations 500 .

Example 5.4 (Rocket car [42]).

$$
\begin{array}{ll}
\text { minimize } & 0.5\left(\left(x_{1}(5)\right)^{2}+\left(x_{2}(5)\right)^{2}\right), \\
\text { subject to } & \dot{x}_{1}(t)=x_{2}(t), \quad \dot{x}_{2}(t)=p(t), \forall t \in[0,5], \\
& x_{1}(0)=6, \quad x_{2}(0)=1, \quad p(t) \in[-1,1] .
\end{array}
$$

The exact optimal control of Example 5.4 is $p^{*}(t)=1$ if $t \in(3.517,5]$ and $p^{*}(t)=-1$ if $t \in(0,3.517]$.

Example 5.5 (see [44]).

$$
\begin{array}{ll}
\operatorname{minimize} & -x_{1}(2)+\left(x_{2}(2)\right)^{2}, \\
\text { subject to } & \dot{x}_{1}(t)=x_{2}(t), \quad \dot{x}_{2}(t)=p(t), \forall t \in[0,2], \\
& x_{1}(0)=0, \quad x_{2}(0)=0, \quad p(t) \in[-1,1] .
\end{array}
$$

The exact optimal control of Example 5.5 is $p^{*}(t)=1$ if $t \in[0,1.2)$ and $p^{*}(t)=-1$ if $t \in(1.2,2]$.

Figure 7 demonstrates the optimal control of the proposed Algorithm (4.2) on Example 5.4 and the proposed Algorithm (4.3) on Example 5.5. The numerical results of all algorithms for Examples 5.4 and 5.5 are shown in Table 4.

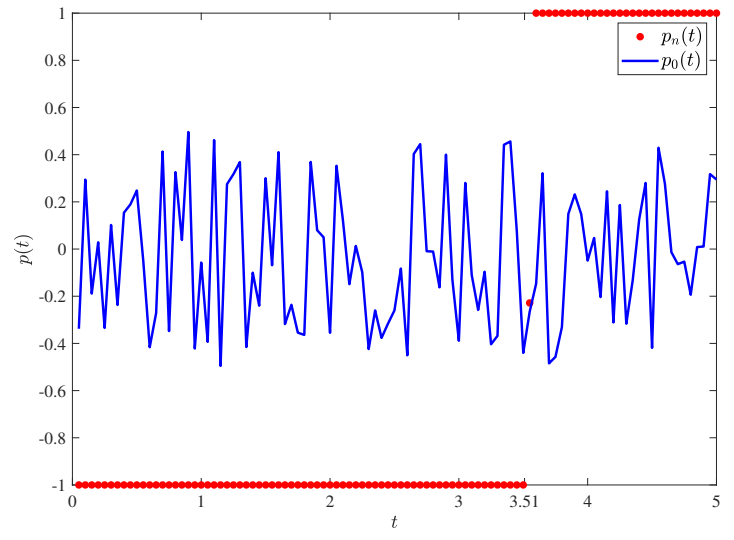

(a) Initial and optimal controls

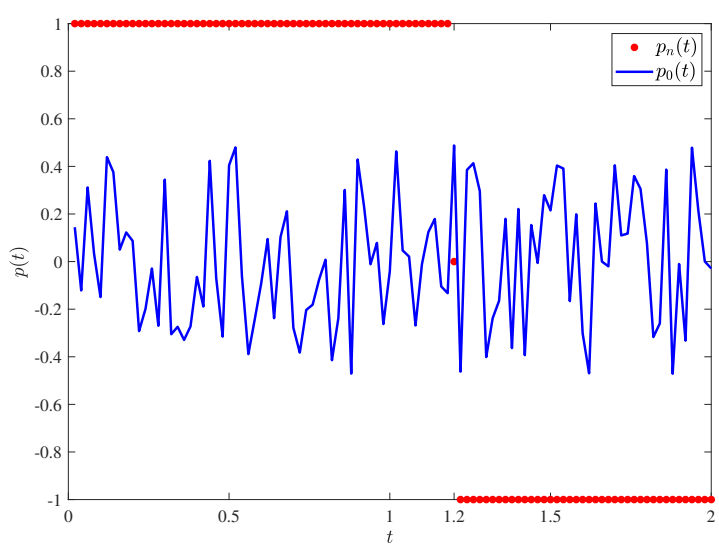

(b) Initial and optimal controls

FIGURE 7. Optimal control of our Algorithm (4.2) on Example 5.4 (Left) and our Algorithm (4.3) on Example 5.5 (Right) 
TABLE 4. Numerical results of all algorithms for Examples 5.4 and 5.5

\begin{tabular}{lcccccc}
\hline \multirow{2}{*}{ Algorithms } & \multicolumn{3}{c}{ Example 5.4 } & \multicolumn{3}{c}{ Example 5.5 } \\
\cline { 2 - 7 } & Iter. & CPU $(s)$ & $D_{n}$ & Iter. & CPU $(s)$ & $D_{n}$ \\
\hline Our Alg. (4.2) & 58 & 0.0273 & $9.5285 \mathrm{E}-05$ & 46 & 0.0338 & $9.7939 \mathrm{E}-05$ \\
Our Alg. (4.3) & 93 & 0.0390 & $9.9943 \mathrm{E}-05$ & 92 & 0.0518 & $9.9112 \mathrm{E}-05$ \\
Our Alg. (4.4) & 103 & 0.0440 & $9.9829 \mathrm{E}-05$ & 102 & 0.0671 & $9.9333 \mathrm{E}-05$ \\
THR Alg. 3.2 & 500 & 0.2428 & $1.4383 \mathrm{E}-02$ & 500 & 0.2540 & $8.6621 \mathrm{E}-03$ \\
TLQ Alg. (3.39) & 500 & 0.1861 & $1.4386 \mathrm{E}-02$ & 500 & 0.1721 & $8.9668 \mathrm{E}-03$ \\
\hline
\end{tabular}

Remark 5.2. It can be seen from Fig. 7 and Table 4 that the proposed Algorithms (4.2)-(4.4) can be used to solve optimal control problems and they perform better than some known algorithms in the literature $[40,41]$.

\section{Conclusions}

In this paper, we proposed three new iterative algorithms to solve the equilibrium problem constrained by the fixed point problem in real Hilbert spaces. The bifunction $f$ in the equilibrium problem is pseudomonotone and the mapping $S$ in the fixed point problem is quasi-nonexpansive or $\lambda$-demicontractive. The proposed algorithms are inspired by the inertial subgradient extragradient method, the viscosity-type method, and the Mann-type method. Our algorithms apply a new non-monotonic step size criterion allowing them to work well without the prior knowledge of the Lipschitz constant of the bifunction $f$. We established the strong convergence of the iterative sequences generated by the proposed algorithms under some suitable conditions. Under the assumption that the bifunction is strongly pseudomonotone, we obtained the linear convergence of a new algorithm. The applications of the suggested iterative schemes to variational inequality problems were discussed. Finally, several numerical examples and applications to optimal control problems demonstrated the computational performance of the proposed algorithms compared to some known ones in the literature. The algorithms proposed in this paper improved and generalized many previously known results in the literature.

\section{Acknowledgments}

The research of the third author was supported by the Grant MOST (108-2115-M-039-005-MY3).

\section{REFERENCES}

[1] E. Blum, W. Oettli, From optimization and variational inequalities to equilibrium problems, Math. Student 63 (1994), 123-145.

[2] K. Fan, A minimax inequality and applications, Inequalities III, Academic Press, New York, 1972.

[3] A. Moudafi, Proximal point algorithm extended to equilibrum problem, J. Nat. Geom. 15 (1999), 91-100.

[4] G. Mastroeni, On auxiliary principle for equilibrium problems, In: Daniele, P., et al. (eds.) Equilibrium Problems and Variational Models, pp. 289-298. Kluwer Academic Publishers, Dordrecht, 2003.

[5] G. Mastroeni, Gap functions for equilibrium problems, J. Global Optim. 27 (2003), 411-426.

[6] L. Liu, X. Qin, Strong convergence theorems for solving pseudo-monotone variational inequality problems and applications, Optimization, doi: 10.1080/02331934.2021.1905641.

[7] P.N. Anh, A hybrid extragradient method extended to fixed point problems and equilibrium problems, Optimization 62 (2013), 271-283. 
[8] P.T. Vuong, J.J. Strodiot, V.H. Nguyen, Extragradient methods and linesearch algorithms for solving Ky Fan inequalities and fixed point problems, J. Optim. Theory Appl. 155 (2012), 605-627.

[9] P.T. Vuong, J.J. Strodiot, V.H. Nguyen, On extragradient-viscosity methods for solving equilibrium and fixed point problems in a Hilbert space, Optimization 64 (2015), 429-451.

[10] G.M. Korpelevich, The extragradient method for finding saddle points and other problems, Èkonom. i Mat. Metody 12 (1976), 747-756.

[11] T.D. Quoc, L.D. Muu, V.H. Nguyen, Extragradient algorithms extended to equilibrium problem, Optimization 57 (2008), 749-776.

[12] V. Dadashi, O.S. Iyiola, Y. Shehu, The subgradient extragradient method for pseudomonotone equilibrium problems, Optimization 69 (2020), 901-923.

[13] D.V. Hieu, A. Gibali, Strong convergence of inertial algorithms for solving equilibrium problems, Optim. Lett. 14 (2020), 1817-1843.

[14] D.V. Hieu, P.K. Quy, L.V. Vy, Explicit iterative algorithms for solving equilibrium problems, Calcolo 56 (2019), Article ID 11.

[15] Y. Shehu, O.S. Iyiola, D.V. Thong, N.T.C. Van, An inertial subgradient extragradient algorithm extended to pseudomonotone equilibrium problems, Math. Meth. Oper. Res. 93 (2021), 213-242.

[16] Y. Censor, A. Gibali, S. Reich, The subgradient extragradient method for solving variational inequalities in Hilbert space, J. Optim. Theory Appl. 148 (2011), 318-335.

[17] Y. Censor, A. Gibali, S. Reich, Strong convergence of subgradient extragradient methods for the variational inequality problem in Hilbert space, Optim. Methods Softw. 26 (2011), 827-845.

[18] Y. Censor, A. Gibali, S. Reich, Extensions of Korpelevich's extragradient method for the variational inequality problem in Euclidean space, Optimization, 61 (2012), 1119-1132.

[19] D.V. Thong, P. Cholamjiak, M.T. Rassias, Y.J. Cho, Strong convergence of inertial subgradient extragradient algorithm for solving pseudomonotone equilibrium problems, Optim. Lett. (2021), doi:10.1007/s11590-02101734-z.

[20] D.V. Hieu, Halpern subgradient extragradient method extended to equilibrium problems, Rev. R. Acad. Cienc. Exactas Fís. Nat. Ser. A Math. RACSAM 111 (2017), 823-840.

[21] M.H. Duc, H.N.T. Thanh, T.T.T. Huyen, B.V. Dinh, The Ishikawa subgradient extragradient method for equilibrium problems and fixed point problems in Hilbert spaces, Numer. Funct. Anal. Optim. 41 (2020), 1065-1088.

[22] D.V. Hieu, New inertial algorithm for a class of equilibrium problems, Numer. Algorithms 80 (2019), 14131436.

[23] D.V. Hieu, B.H. Thai, P. Kumam, Parallel modified methods for pseudomonotone equilibrium problems and fixed point problems for quasi-nonexpansive mappings, Adv. Oper. Theory 5 (2020), 1684-1717.

[24] L.O. Jolaoso, T.O. Alakoya, A. Taiwo, O.T. Mewomo, Inertial extragradient method via viscosity approximation approach for solving equilibrium problem in Hilbert space, Optimization 70 (2021), 387-412.

[25] J. Yang, H. Liu, The subgradient extragradient method extended to pseudomonotone equilibrium problems and fixed point problems in Hilbert space, Optim. Lett. 14 (2020), 1803-1816.

[26] D.V. Hieu, J.J. Strodiot, L.D. Muu, Strongly convergent algorithms by using new adaptive regularization parameter for equilibrium problems, J. Comput. Appl. Math. 376 (2020), Article ID 112844.

[27] B. Polyak, Some methods of speeding up the convergence of iteration methods, USSR Comput. Math. Math. Phys. 4 (1964), 1-17.

[28] F. Alvarez, H. Attouch, An inertial proximal method for maximal monotone operators via discretization of a nonlinear oscillator with damping, Set-Valued Anal. 9 (2001), 3-11.

[29] J. Fan, L. Liu, X. Qin, A subgradient extragradient algorithm with inertial effects for solving strongly pseudomonotone variational inequalities, Optimization 69 (2020), 2199-2215.

[30] Q.-L. Dong, Y.J. Cho, L.L. Zhong, T.M. Rassias, Inertial projection and contraction algorithms for variational inequalities, J. Global Optim. 70 (2018), 687-704.

[31] Z. Zhou, B. Tan, S. Li, An accelerated hybrid projection method with a self-adaptive step-size sequence for solving split common fixed point problems, Math. Methods Appl. Sci. 44 (2021), 7294-7303.

[32] J.M. Ortega, W.C. Rheinboldt, Iterative Solution of Nonlinear Equations in Several Variables, SIAM, Philadelphia, 2000. 
[33] J.V. Tiel, Convex Analysis: An Introductory Text, Wiley, New York, 1984.

[34] H.H. Bauschke, P.L. Combettes, Convex Analysis and Monotone Operator Theory in Hilbert Spaces, Springer, New York, 2011.

[35] P.E. Maingé, A hybrid extragradient-viscosity method for monotone operators and fixed point problems, SIAM J. Control Optim. 47 (2008), 1499-1515.

[36] H.K. Xu, Iterative algorithms for nonlinear operators, J. Lond. Math. Soc. 66 (2002), 240-256.

[37] D.V. Thong, D.V Hieu, Modified subgradient extragradient algorithms for variational inequality problems and fixed point problems, Optimization 67 (2018), 83-102.

[38] S. Boyd, L. Vandenberghe, Convex Optimization, Cambridge University Press, Cambridge, 2004.

[39] I. Yamada, N. Ogura, Hybrid steepest descent method for variational inequality operators over the problem certain fixed point set of quasi-nonexpansive mappings, Numer. Funct. Anal. Optim. 25 (2004), 619-655.

[40] B. Tan, L. Liu, X. Qin, Self adaptive inertial extragradient algorithms for solving bilevel pseudomonotone variational inequality problems, Jpn. J. Ind. Appl. Math. 38 (2021), 519-543.

[41] D.V. Thong, D.V. Hieu, T.M Rassias, Self adaptive inertial subgradient extragradient algorithms for solving pseudomonotone variational inequality problems, Optim. Lett. 14 (2020), 115-144.

[42] J. Preininger, P.T. Vuong, On the convergence of the gradient projection method for convex optimal control problems with bang-bang solutions, Comput. Optim. Appl. 70 (2018), 221-238.

[43] P.T. Vuong, Y. Shehu, Convergence of an extragradient-type method for variational inequality with applications to optimal control problems, Numer. Algorithms 81 (2019), 269-291.

[44] B. Bressan, B. Piccoli, Introduction to the Mathematical Theory of Control. American Institute of Mathematical Sciences, San Francisco, 2007. 